\title{
Identification of drought-responsive miRNAs and physiological characterization of tea plant (Camellia sinensis L.) under drought stress
}

Yuqiong Guo ${ }^{1}$, Shanshan Zhao ${ }^{1}$, Chen Zhu' ${ }^{1}$ Xiaojun Chang ${ }^{1}$, Chuan Yue ${ }^{1}$, Zhong Wang ${ }^{1}$, Yuling Lin ${ }^{1,2}$ and Zhongxiong Lai ${ }^{1,2^{*}}$

\begin{abstract}
Background: Drought stress is one of the major natural challenges in the main tea-producing regions of China. The tea plant (Camellia sinensis) is a traditional beverage plant whose growth status directly affects tea quality. Recent studies have revealed that microRNAs (miRNAs) play key functions in plant growth and development. Although some miRNAs have been identified in C. sinensis, little is known about their roles in the drought stress response of tea plants.

Results: Physiological characterization of Camellia sinensis 'Tieguanyin' under drought stress showed that the malondialdehyde concentration and electrical conductivity of leaves of drought-stressed plants increased when the chlorophyll concentration decreased under severe drought stress. We sequenced four small-RNA (sRNA) libraries constructed from leaves of plants subjected to four different treatments, normal water supply (CK); mild drought stress (T1); moderate drought stress (T2) and severe drought stress (T3). A total of 299 known mature miRNA sequences and 46 novel miRNAs were identified. Gene Ontology enrichment analysis revealed that most of the differentially expressed-miRNA target genes were related to regulation of transcription. Kyoto Encyclopedia of Genes and Genomes analysis revealed that the most highly enriched pathways under drought stress were D-alanine metabolism, sulfur metabolism, and mineral absorption pathways. Real-time quantitative PCR (qPCR) was used to validate the expression patterns of 21 miRNAs (2 up-regulated and 19 down-regulated under drought stress). The observed co-regulation of the miR166 family and their targets ATHB-14-like and ATHB-15like indicate the presence of negative feedback regulation in miRNA pathways.

Conclusions: Analyses of drought-responsive miRNAs in tea plants showed that most of differentially expressedmiRNA target genes were related to regulation of transcription. The results of study revealed that the expressions of phase-specific miRNAs vary with morphological, physiological, and biochemical changes. These findings will be useful for research on drought resistance and provide insights into the mechanisms of drought adaptation and resistance in C. sinensis.
\end{abstract}

Keywords: Camellia sinensis, Drought stress, miRNA, Physiological characteristics

\footnotetext{
*Correspondence: laizx01@163.com

${ }^{1}$ College of Horticulture, Fujian Agriculture and Forestry University, Fuzhou 350002, China

${ }^{2}$ Institute of Horticultural Biotechnology, Fujian Agriculture and Forestry University, Fuzhou 350002, China
} International License (http://creativecommons.org/licenses/by/4.0/), which permits unrestricted use, distribution, and reproduction in any medium, provided you give appropriate credit to the original author(s) and the source, provide a link to the Creative Commons license, and indicate if changes were made. The Creative Commons Public Domain Dedication waiver (http://creativecommons.org/publicdomain/zero/1.0/) applies to the data made available in this article, unless otherwise stated. 


\section{Background}

Tea (Camellia sinensis L.) is an economically important crop from southwestern China whose leaves are the source of one of the most popular non-alcoholic beverages worldwide. Tea leaf quality and yield mainly depend on the growth status of tea plants. In the main teaproducing regions of China, which are spread over the middle and lower reaches of the Yangtze River, tea trees are under drought stress during the tea production period, especially in summer and fall. Drought stress has been reported to reduce tea production by $14 \%-33 \%$ and to increase tea plant mortality by $6 \%-19 \%$ [1]. It is urgent, therefore, to determine how tea plants respond to drought stress.

Increasing attention is being paid to the drought tolerance of tea plants. Several studies have found that tea plants adapt to drought stress by various morphological changes, osmotic regulation, reactive oxygen species scavenging, and plant hormone regulation [2-4]. In addition, amplified fragment length polymorphism (AFLP) and suppression subtractive hybridization (SSH) analyses have been used to identify genes related to the drought response of $C$. sinensis [5-7]. Transcriptome analyses have been used to investigate gene regulation under drought conditions, resulting in the identification of many genes involved in the drought response [8,9]. Despite these advances, there is still much to learn about the regulation of the plant drought response.

Apart from the series of physiological changes mentioned above, miRNAs have been shown to be involved in drought stress responses in some plants. However, the miRNA-associated regulatory networks in C. sinensis remain to be uncovered. Hence, the response of miRNAs in $C$. sinensis to stress and their roles in adaptation and tolerance have become major research topics [10-12]. Plant miRNAs are known have important functions in responses to biotic and abiotic stresses [13-24]. Studies have shown that miRNAs function as important modulators of drought tolerance by influencing the cleavage of drought-responsive genes or inhibiting their translation. Many of these miRNAs target genes encoding transcription factors; therefore, miRNAs function at the center of drought-stress regulatory networks [25-27]. Thus, the identification of miRNAs and their target genes is essential to reveal the molecular mechanism of miRNAs in the drought stress response.

Numerous conserved miRNAs and their targets have recently been identified in C. sinensis [28-30]. Some of these miRNAs are responsive to dormancy [29] and cold stress [30]. Using small RNA (sRNA) sequencing, Liu and $\mathrm{Xu}$ et al. [31] identified 268 conserved and 62 novel miRNAs from the drought-resistant tea cultivar 'Ningzhou2' and the drought-susceptible tea cultivar 'Zhuyeqi'. While there is a large amount of information on miRNA expression under drought stress in other woody plants, relatively little information is available for tea plants. In the present study, we used Illumina HiSeq 2500 technology to identify putative miRNAs and investigated their expression profiles in the unique oolong tea cultivar 'Tieguanyin' under four drought stress conditions. We predicted the target genes of the miRNAs related to the drought stress response in $C$. sinensis. We also analyzed physiological characteristics of drought-stressed and control plants, including malondialdehyde (MDA) concentration, electrical conductivity, chlorophyll concentration, and leaf water content. Our results provide new information about the regulatory mechanism(s) of miRNAs in the drought stress response of tea plants.

\section{Methods}

Plant materials and drought stress treatments

The experimental material was C. sinensis 'Tieguanyin', the most well-known oolong tea cultivar. Healthy 1year-old tea plant cuttings were transplanted into pots and grown in a greenhouse under a 12-h light $\left(30{ }^{\circ} \mathrm{C}\right) /$ 12 -h dark $\left(20{ }^{\circ} \mathrm{C}\right)$ photoperiod and $40 \%-65 \%$ relative humidity. Drought treatments were applied using the compensatory watering method, with all other ambient conditions held constant. There were four levels of drought treatment (Fig. 1a): normal water supply (soil moisture content $=19.50 \%$; CK), mild drought stress (soil moisture content $=15.20 \%$; T1), moderate drought stress (soil moisture content $=10.17 \%$; T2) and severe drought stress (soil moisture content $=5.54 \%$; T3). Each treatment lasted for 10 days. In addition, three independent biological replicates were established for each drought treatment. Each replicate was collected from 10 randomly selected tea plants. The tender leaves of $C$. sinensis were sampled, frozen immediately in liquid nitrogen, and stored at $-80{ }^{\circ} \mathrm{C}$ until further analysis.

\section{Determination of relative water content, electrical conductivity, and chlorophyll and MDA concentrations}

Leaves were randomly sampled from plants in the drought treatments and their physiological characteristics were measured. To detect the effects of drought stress on tea plants, relative water contents (including soil moisture and leaf water content) were determined according to Upadhyaya et al. [32]. The leaf chlorophyll concentration was determined as previously described [33]; MDA was extracted and analyzed as described elsewhere [34], and leaf conductivity was measured as described by Luo et al. [35].

\section{Total RNA extraction}

For miRNA sequencing, total RNA was extracted from tea leaves using a Trizol Reagent kit (Invitrogen/Life Technologies, Carlsbad, CA, USA) according to the 

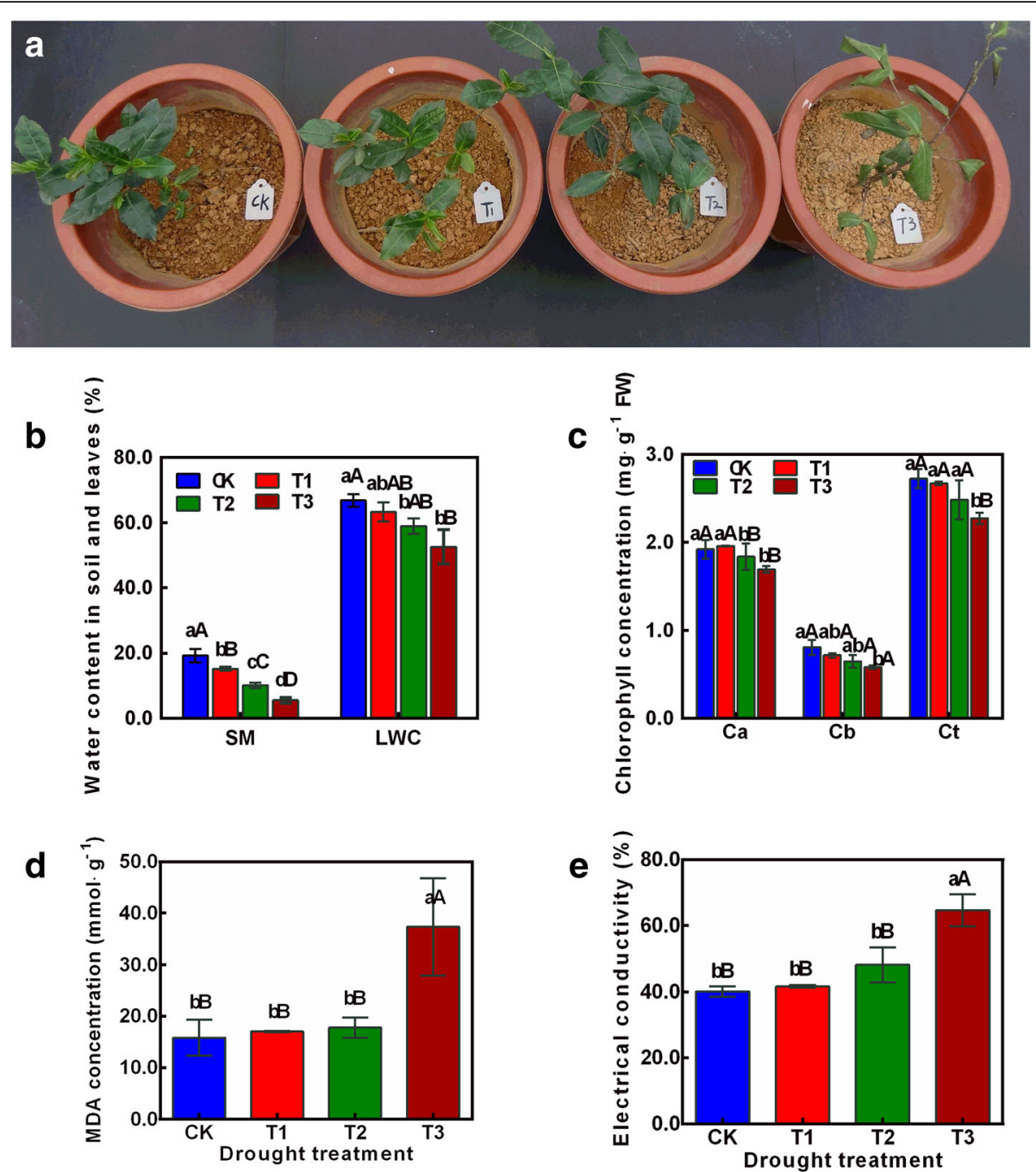

Fig. 1 Physiological changes in Camellia sinensis 'Tieguanyin' plants subjected to different drought stress conditions. a Phenotypes of tea plants after exposure to drought stress for 10 days. CK: normal water supply; T1: mild drought stress; T2: moderate drought stress; T3: severe drought stress. b-e Soil moisture (SM) and leaf water content (LWC) (b), chlorophyll a (Ca), chlorophyll b (Cb), and total chlorophyll (Ct) concentration (c), malondialdehyde (MDA) concentration (d), and electrical conductivity (e) of tea plants at different drought stress stages. Data are means \pm SE $(n=3)$

manufacturer's instructions. Total RNA quality was checked by $1 \%$ agarose gel electrophoresis and ultra micro-ultraviolet spectrophotometry. The RNA samples were stored at $-80{ }^{\circ} \mathrm{C}$ until miRNA sequencing.

\section{Library preparation and sequencing}

The four small RNA libraries generated respectively from four treated samples (CK, T1, T2 and T3) and sequenced by the Biomarker Technology Co. (Beijing, China, http://www.biomarker.com.cn/) using an Illumina HiSeq 2500 instrument. Total RNA was extracted from samples from each treatment, and linkers were added to the 5' and 3' ends of RNA by T4 RNA ligase. Then, the target fragments were amplified by reverse transcription PCR using synthesized first-strand cDNA as the template, and screened by polyacrylamide gel electrophoresis. Each small RNA library was constructed using the fragments reclaimed from the gel. Finally, each small RNA library was sequenced.

The candidate miRNA genes selected for further investigation were those that satisfied the following criteria, based on Meyers et al. [36]: (1) a mature sequence localized in one arm of the stem-loop structure and between 19 and 24 nt in length; (2) corresponding miRNA* sequence identified; (3) premiRNA sequence folded into an appropriate stemloop hairpin secondary structure; (4) minimum free energy (MFE) of secondary structures $\leq-20 \mathrm{kcal} / \mathrm{mol}$; and (5) no more than $4 \mathrm{nt}$ mismatches in the miRNA:miRNA* duplex. 


\section{MiRNA annotation and target gene prediction}

To identify sRNAs originating from miRNA degradation fragments, exon and intron sense and antisense sequences were compared between sRNA and miRNAs using Overlap software. The identified sRNA sequences were successfully annotated using rRNA, scRNA, snoRNA, snRNA, and tRNA sequences from Rfam 3 and GenBank databases. To ensure unique sRNA annotations, sequence comparisons were prioritized in the following order: rRNAs $>$ conserved miRNAs $>$ repeats $>$ exons > introns.

The potential target genes of novel $C$. sinensis miRNAs were predicted using the algorithms of Allen [37] and Schwab [38], with the following criteria: (1) less than four mismatches between the sRNA and its target (mismatches in G-U bases were counted as 0.5); (2) less than two adjacent mismatches in the miRNA/target duplex; (3) less than 2.5 mismatches in positions 1-12 of the miRNA/target duplex ( $5^{\prime}$ end of the miRNA); (4) no adjacent mismatches in positions 2-12 of the miRNA/target duplex ( $5^{\prime}$ end of the miRNA); (5) no mismatches in positions $10-11$ of the miRNA/target duplex; and (6) minimum free energy of the miRNA/target duplex $\geq 75 \%$ of the minimum free energy of the miRNA bound to its optimal complement.

\section{Real-time quantitative PCR (qPCR) detection of miRNAs and their targets in $C$. sinensis}

The expression levels of relevant miRNAs and their target genes were analyzed by qPCR. The cDNA of miRNAs was synthesized using a SYBR One Step PrimeScript miRNA cDNA Synthesis kit (Takara, Otsu, Japan, Code: RR716). The qPCR analyses were conducted using SYBR Premix Ex Taq (Takara) on a LightCycler480 qPCR instrument (Roche Applied Sciences, Basel, Switzerland) under the following cycling conditions: preheating at $94{ }^{\circ} \mathrm{C}$ for $10 \mathrm{~s}$, followed by 50 cycles of $94{ }^{\circ} \mathrm{C}$ for $5 \mathrm{~s}, 57-62{ }^{\circ} \mathrm{C}$ for $15 \mathrm{~s}$ and $72{ }^{\circ} \mathrm{C}$ for $10 \mathrm{~s}$, with a final step at $40{ }^{\circ} \mathrm{C}$ for $30 \mathrm{~s}$. A melting curve analysis was performed to check for primer dimers. The primers used for $\mathrm{qPCR}$ are listed in Additional file 1: Table S1. All reactions were repeated in triplicate, with the 18S rRNA gene used as an internal control. The relative expression levels of miRNAs were calculated using the $2^{-\Delta \Delta C t}$ method of Schmittgen et al. [39].

\section{Statistical analysis}

Statistical analyses were conducted using SPSS version 13 (SPSS, Inc., Chicago, IL, USA). The data were analyzed by one-way analysis of variance followed by Tukey's post-hoc test, with differences considered significant at $p<0.05$.

\section{Results}

Changes in chlorophyll and MDA concentrations, and electrical conductivity under drought stress in $C$. sinensis The phenotypes of $C$. sinensis leaves were recorded under conditions ranging from normal water supply to severe drought (CK, T1, T2, and T3). The leaves were slightly curled and deformed under T1, and dull, curled, and wilted under T2. With increasing severity of drought stress (T3), leaves wilted further and most branches died (Fig. 1a). The leaf water content (LWC), soil moisture (SM), chlorophyll and MDA concentrations, and electrical conductivity were measured under the four different treatments. The LWC may reflect metabolic intensity and, to some extent, indicate the water absorption capacity and dehydration tolerance under drought conditions. Compared with the LWC in CK, that in T1, T2, and T3 was decreased by $6.93 \%$, $11.18 \%$, and $18.86 \%$, respectively. Chlorophyll a, chlorophyll b, and total chlorophyll concentrations decreased slightly, but differences among treatments were not significant (Fig. 1c). The MDA concentration and leaf electrical conductivity were markedly higher under the T3 treatment than under the other treatments (Fig. 1d-e).

\section{Construction and high-throughput sequencing of sRNA li- braries from $C$. sinensis}

To identify drought-responsive miRNAs in tea plants, four sRNA libraries generated from plants in the CK, T1, T2, and T3 treatments were sequenced using the Illumina platform. Table 1 provides a statistical summary of sequencing results for the four sRNA libraries.

After removing 5'-adapters, 3'-adapters, and lowquality reads, we obtained 15,777,773, 13,218,397, $14,510,362$, and $13,452,128$ clean reads from the CK, T1, T2, and T3 samples, respectively. The proportion of clean reads out of total sequencing reads was $99.86 \%$, 99.58\%, 99.58\%, and $98.95 \%$ for CK, T1, T2, and T3, respectively, indicating that the read quality was sufficient for further analyses.

As shown in Fig. 2a, the lengths of all C. sinensis sRNA sequences ranged between 21 and $24 \mathrm{nt}$. As the severity of drought stress increased, the number of 21-nt sRNA sequences increased (by $3.97 \%, 4.52 \%$, and $22 \%$ in $\mathrm{T} 1$, T2, and T3, respectively, compared with CK) while the number of 24-nt sRNAs decreased.

\section{Classification and annotation of sRNAs under drought stress in $C$. sinensis}

Comparison with precursor miRNAs and mature miRNAs of all plants in miRBase 21 (http://www.mirbase.org/) revealed that $32,364(0.37 \%), 29,770$ (0.41\%), 35,077 (0.45\%), and $30,580(0.59 \%)$ unique sequences from CK, T1, T2, and T3, respectively, were similar to known miRNAs [40]. Other unique sequences, such as rRNAs (CK: 37,966, 
Table 1 Summary of sequencing results of small RNA libraries constructed from Tieguanyin tea plants subjected to normal water supply (CK), mild drought stress (T1), moderate drought stress (T2) and severe drought stress (T3) treatments

\begin{tabular}{lllll}
\hline Category & CK & T1 & T2 & T3 \\
& Reads No. & Reads No. & Reads No. & Reads No. \\
& (Percentage) & (Percentage) & (Percentage) & (Percentage) \\
\hline Total_reads & $15,843,812$ & $13,383,907$ & $14,689,202$ & $13,704,671$ \\
High_quality & $15,799,925$ & $13,273,561$ & $14,569,424$ & $13,594,243$ \\
3'adapter_null & $(100 \%)$ & $(100 \%)$ & $(100 \%)$ & $(100 \%)$ \\
& 3287 & 10,422 & 11,340 & 8303 \\
Insert_null & $(0.02 \%)$ & $(0.08 \%)$ & $(0.08 \%)$ & $(0.06 \%)$ \\
5'adapter_contaminants & 1774 & 2598 & 2888 & 9478 \\
& $(0.01 \%)$ & $(0.02 \%)$ & $(0.02 \%)$ & $(0.07 \%)$ \\
Smaller_than 18nt & $(0.07 \%)$ & $(0.21 \%)$ & $(0.22 \%)$ & $(0.58 \%)$ \\
& 3008 & 10,268 & 9309 & 42,893 \\
PolyA & $(0.02 \%)$ & $(0.08 \%)$ & $(0.06 \%)$ & $(0.32 \%)$ \\
Clean_reads & 3688 & 3372 & 3630 & 2612 \\
& $(0.02 \%)$ & $(0.03 \%)$ & $(0.02 \%)$ & $(0.02 \%)$ \\
& $15,777,773$ & $13,218,397$ & $14,510,362$ & $13,452,128$ \\
& $(99.86 \%)$ & $(99.58 \%)$ & $(99.59 \%)$ & $(98.95 \%)$ \\
\hline
\end{tabular}

0.43\%; T1: 56,302, 0.77\%; T2: 55,779, 0.71\%; T3: 120,595, 2.33\%), snRNAs (CK: 1969, 0.02\%; T1: 2062, 0.03\%; T2: 2363, 0.03\%; T3: 3289, 0.06\%), snoRNAs (CK: 685, 0.01\%; T1: 745, 0.01\%; T2: 883, 0.01\%; T3: 892, 0.02\%), and tRNAs (CK: 2632, 0.03\%; T1: 4201, 0.06\%; T2: 4291, 0.05\%; T3: 8184, 0.16\%), were also characterized according to the Rfam database (http://rfam.xfam.org/) [41]. The distribution of characterized sequences from the four libraries is shown in Table 2 .

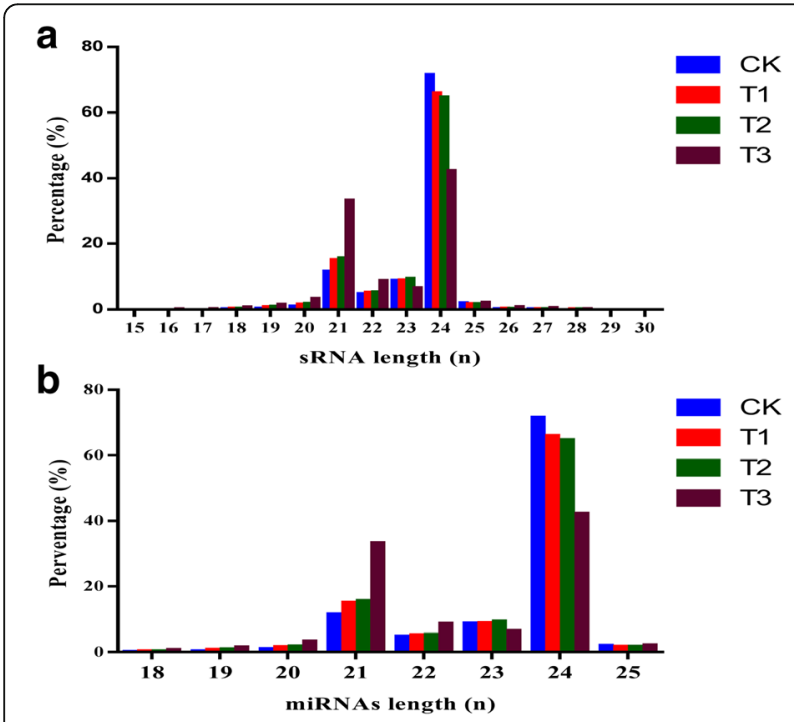

Fig. 2 Size distributions of sRNAs (a) and miRNAs (b) in Camellia sinensis plants under different drought stress conditions

\section{Identification of conserved miRNAs during drought stress} in C. sinensis

A total of 191 known mature miRNAs were identified in C. sinensis: 117 from CK, 127 from T1, 127 from T2, and 129 from T3, with 103 common to all four libraries. The number of miRNA target genes predicted in CK, T1, T2, and T3 was 2078, 2077, 2778, and 2064, respectively (Table 3). In total, 4376 target genes of 191 conserved miRNAs were obtained from the four treatment samples.

Although the lengths of known C. sinensis miRNAs ranged from 18 to $25 \mathrm{nt}$, most were 24-nt (CK: 71.48\%; T1: 65.87\%; T2: 64.63\%; T3: 42.26\%) or 21-nt (CK: 11.50\%; T1: 15.02\%; T2: 15.57\%; T3: 33.25\%) (Fig. 2b). As shown in Fig. 3, the majority of miRNA sequences from the $C$. sinensis libraries started with uridine. The characteristics of $C$. sinensis miRNA sequences were consistent with those previously reported for miRNA sequences [42].

Table 2 Distribution of unique small RNA (sRNA) sequences from Tieguanyin tea plants subjected to normal water supply (CK), mild drought stress (T1), moderate drought stress (T2) and severe drought stress (T3) treatments. "Unann" refers to sRNA sequences for which no annotation information could be obtained by database comparisons

\begin{tabular}{|c|c|c|c|c|}
\hline \multirow[t]{3}{*}{ Types } & CK & $\mathrm{T} 1$ & $\mathrm{~T} 2$ & $\mathrm{~T} 3$ \\
\hline & UniquesRNAs & Unique sRNAs & Unique sRNAs & Unique sRNAs \\
\hline & (Percentage) & (Percentage) & (Percentage) & (Percentage) \\
\hline \multirow[t]{2}{*}{ Total } & $8,797,521$ & $7,277,866$ & $7,842,237$ & $5,165,455$ \\
\hline & $(100 \%)$ & $(100 \%)$ & $(100 \%)$ & $(100 \%)$ \\
\hline \multirow[t]{2}{*}{ Exon_antisense } & 743 & 842 & 897 & 1071 \\
\hline & $(0.01 \%)$ & $(0.01 \%)$ & $(0.01 \%)$ & $(0.02 \%)$ \\
\hline \multirow[t]{2}{*}{ Exon_sense } & 1086 & 1354 & 1443 & 2136 \\
\hline & $(0.01 \%)$ & $(0.02 \%)$ & $(0.02 \%)$ & $(0.04 \%)$ \\
\hline \multirow[t]{2}{*}{ Intron_antisense } & 156 & 180 & 164 & 227 \\
\hline & $(0 \%)$ & $(0 \%)$ & $(0 \%)$ & $(0 \%)$ \\
\hline \multirow[t]{2}{*}{ Intron_sense } & 189 & 211 & 205 & 287 \\
\hline & $(0 \%)$ & $(0 \%)$ & $(0 \%)$ & $(0 \%)$ \\
\hline \multirow[t]{2}{*}{ miRNA } & 32,364 & 29,770 & 35,077 & 30,580 \\
\hline & $(0.37 \%)$ & $(0.41 \%)$ & $(0.45 \%)$ & $(0.59 \%)$ \\
\hline \multirow[t]{2}{*}{ rRNA } & 37,966 & 56,302 & 55,779 & 120,595 \\
\hline & $(0.43 \%)$ & $(0.77 \%)$ & $(0.71 \%)$ & $(2.33 \%)$ \\
\hline \multirow[t]{2}{*}{ Repeat } & 31,260 & 28,218 & 29,298 & 26,544 \\
\hline & $(0.36 \%)$ & $(0.39 \%)$ & $(0.37 \%)$ & $(0.51 \%)$ \\
\hline \multirow[t]{2}{*}{ snRNA } & 1969 & 2062 & 2363 & 3289 \\
\hline & $(0.02 \%)$ & $(0.03 \%)$ & $(0.03 \%)$ & $(0.06 \%)$ \\
\hline \multirow[t]{2}{*}{ snoRNA } & 685 & 745 & 883 & 892 \\
\hline & $(0.01 \%)$ & $(0.01 \%)$ & $(0.01 \%)$ & $(0.02 \%)$ \\
\hline \multirow[t]{2}{*}{ tRNA } & 2632 & 4201 & 4291 & 8184 \\
\hline & $(0.03 \%)$ & $(0.06 \%)$ & $(0.05 \%)$ & $(0.16 \%)$ \\
\hline \multirow[t]{2}{*}{ unann } & $8,688,471$ & $7,153,981$ & $7,711,837$ & $4,971,650$ \\
\hline & (98.76\%) & (98.30\%) & (98.34\%) & $(96.25 \%)$ \\
\hline
\end{tabular}


Table 3 Number of predicted target genes of conserved and novel miRNAs and novel miRNA precursor candidates. Each entry in the row labeled "Novel miRNAs No." refers to the total number of novel miRNAs followed by the number of novel miRNAs with predicted target gene loci

\begin{tabular}{llllll}
\hline Samples & CK & T1 & T2 & T3 & Total \\
\hline Conserved miRNAs No. & 117 & 127 & 127 & 129 & 191 \\
$\begin{array}{l}\text { Predicted target genes No. of } \\
\text { conserved miRNAs }\end{array}$ & 2078 & 2077 & 2778 & 2064 & 4376 \\
Novel miRNAs No. & $34 /$ & $61 /$ & $46 /$ & $57 /$ & $87 /$ \\
& 29 & 48 & 43 & 52 & 59 \\
$\begin{array}{l}\text { Predicted target genes No. of novel } \\
\text { miRNAs }\end{array}$ & 1535 & 1341 & 2597 & 757 & 5528 \\
& & & & &
\end{tabular}

The percentages of adenine (A), uracil (U), guanine $(G)$, and cytosine $(\mathrm{C})$ at each locus of conserved miRNAs changed dynamically with increasing drought stress in Tieguanyin tea plants (Fig. 3). One of the most frequent bases in C. sinensis conserved miRNAs, G, was present mainly at nucleotide positions $3,4,9,10$, and 22 , while $U$ was found mainly at positions $1,12,13,16$, and 17 . Another heavily represented base in conserved miRNAs was $C$, which was mainly distributed at positions $2,6,7,11,14$, and 18-21.

\section{Prediction of novel miRNAs during drought stress in $C$. sinensis}

To predict novel miRNAs, the Arabidopsis thaliana genome (ftp://ftp.arabidopsis.org/home/tair/Genes/TAIR10_ genome_release) was selected as a reference. Comparisons

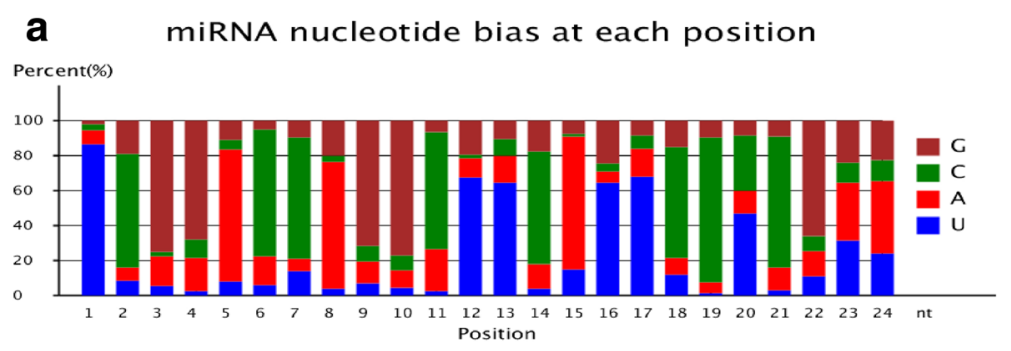

b

miRNA nucleotide bias at each position

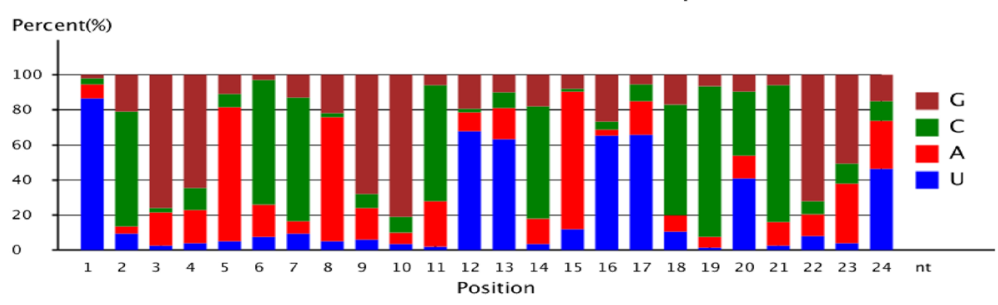

C miRNA nucleotide bias at each position
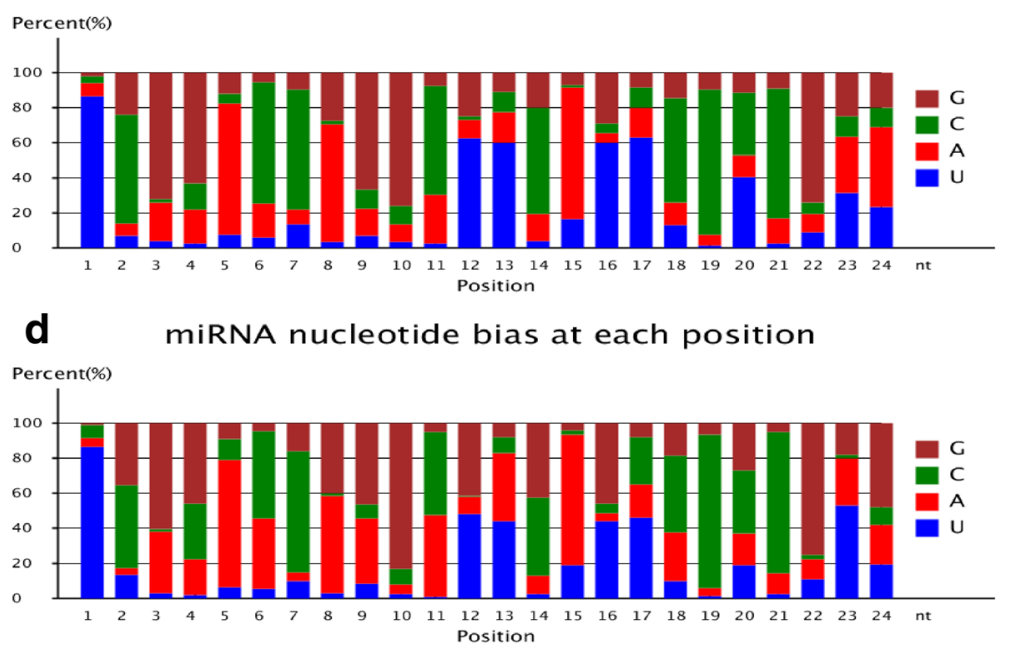

Fig. 3 Nucleotide bias at each position of Camellia sinensis conserved miRNAs under different drought stress conditions. Drought stress conditions were as follows: a normal water supply, $\mathbf{b}$ mild drought stress, $\mathbf{c}$ moderate drought stress, and $\mathbf{d}$ severe drought stress. X-axis represents miRNA nucleotide positions; $y$-axis indicates percentage of each specific nucleotide at each nucleotide position. Brown, green, red, and blue correspond to guanine $(G)$, cytosine $(C)$, adenine $(A)$, and uracil $(U)$, respectively 
of the sRNA data with those in the miRBase and other databases revealed 34, 61, 46, and 57 novel miRNAs from the CK, T1, T2, and T3 sample libraries, respectively. A total of 87 novel miRNAs were identified from the four Tieguanyin tea plant libraries, and 1535, 1341, 2597, and 757 putative target genes of $29,48,43$, and 52 novel miRNAs were identified in the CK, T1, T2, and T3 libraries, respectively. Overall, 5528 target genes of 59 novel miRNAs were predicted (Table 3 ).

The length range of novel miRNAs in the CK, T1, T2, and T3 libraries was 21-23, 20-22, 21-23, and 2023 nt, respectively (Fig. 4). No novel 24-nt miRNAs were identified. Most novel miRNAs in C. sinensis were 21-nt: $7412(57.92 \%)$ in CK, $7790(63.23 \%)$ in $\mathrm{T} 1,9291$ (65.06\%) in T2, and 22,876 (82.71\%) in T3.

To identify additional tea plant miRNAs, we used transcriptome data of $C$. sinensis Tieguanyin as a reference. Hierarchical indexing for spliced alignment of transcripts (Hisat) was used to calculate the coverage of the tea transcriptome data relative to the whole tea genomic data. Calculations were performed as described by Kim et al. [43]. The coverage percentage was $83.80 \%$. According to the sequencing quality of the reference genome, generally more than $70 \%$ coverage is acceptable [43]. Using these analyses, we identified 176 miRNA sequencing reads and predicted 4067 target gene loci (Additional file 2: Table S2).

\section{Analysis of differentially expressed miRNAs during drought stress in C. sinensis}

The expression levels of $C$. sinensis miRNAs differed significantly among the drought treatments (Fig. 5; Additional file 3: Figure S1). In total, we identified 299 known mature miRNA sequences and 46 novel miRNAs. Of these known miRNAs, 101 were differentially expressed between CK and T1 (48 up-regulated and 53 down-regulated; Additional file 4: Table S3), and 108 between CK and T2 (55 up-regulated and 53 downregulated; Additional file 5: Table S4). There were 211 differentially expressed miRNAs between $\mathrm{CK}$ and T3 (112 up-regulated and 99 down-regulated; Additional file 6: Table S5); 76 known miRNAs expressed only in CK, 61 expressed only in T3, 51 up-regulated in T3 compared with CK, and 23 down-regulated in T3 compared with CK.

\section{Gene ontology (GO) and Kyoto encyclopedia of genes and genomes (KEGG) pathway analyses of miRNAs expressed under drought stress in $C$. sinensis}

Next, GO annotations were obtained from the Gene Ontology (http://www.geneontology.org/) and NCBI (ftp://ftp.ncbi.nih.gov/gene/DATA) databases. Gene annotations and classifications according to GO biological process, cellular component, and molecular function categories are provided in Fig. 6a, Additional file 7: Figure S2, Additional file 8: Figure S3, and Fig. 6b. The GO classifications statistics are provided in Additional file 9: Table S6. In these analyses, we assigned 6143, 6264, 6910, and 5389 unigenes in the CK, T1, T2, and T3 libraries, respectively. The top three subgroups in the biological process category were cellular process (1004 genes), metabolic process (968 genes), and single-organism process (607 genes). In the molecular function category, the top three subgroups were binding (1053 genes), catalytic activity (668 genes), and nucleic acid binding transcription factor activity (297 genes). The top three subgroups in the cellular component category were cell, cell part, and organelle, which accounted for $77.78 \%$, $77.56 \%, 77.54 \%$, and $75.13 \%$ of all unigenes in the CK, $\mathrm{T} 1, \mathrm{~T} 2$, and T3 libraries, respectively.

The results of KEGG analysis of miRNAs are shown in Additional file 10: Table S7. The top 20 enriched pathways are shown in Additional file 11: Table S8 and some KEGG scatter diagrams and pathways are shown in Fig. 7 and Additional file 12: Figure S4. The top five pathways based on KEGG enrichment factors were as follows: Dalanine metabolism, sulfur relay system, sulfur metabolism, ascorbate and aldarate metabolism, and mineral absorption for CK vs. T1; D-alanine metabolism, sulfur metabolism, mineral absorption, ascorbate and aldarate metabolism, and alpha-linolenic acid metabolism for CK vs. T2; D-alanine metabolism, mineral absorption, photo transduction, sulfur relay system, and porphyrin and chlorophyll metabolism for CK vs. T3; D-alanine metabolism, lipoic acid metabolism, mineral absorption, sulfur metabolism, and porphyrin and chlorophyll metabolism for T1 vs. T2; D-alanine metabolism, mineral absorption, photo transduction, porphyrin and chlorophyll metabolism, and $\mathrm{Ca}^{2+}$ signaling pathway for T2 vs. T3; and Dalanine metabolism, mineral absorption, photo transduction, porphyrin and chlorophyll metabolism, and sulfur relay system for T1 vs. T3. The most heavily enriched pathway in all groups was D-alanine metabolism, suggesting that this process plays an essential role in the drought stress response. The differential expression of sulfur metabolism (including the sulfur relay system) and mineral absorption pathways in all groups implied these pathways also play key roles in the drought stress response. Metabolic pathways related to D-alanine metabolism, sulfur metabolism, and mineral absorption were significantly affected when tea plants were subjected to mild and moderate drought stress (T1 and T2) compared with CK. Metabolic pathways related to photosynthesis were significantly affected under severe drought stress (T3), suggesting that only severe drought stress significantly affects photosynthesis in tea plants. Liang [44] found that drought stress increased D-alanine synthase activity in tobacco. Sulfur 


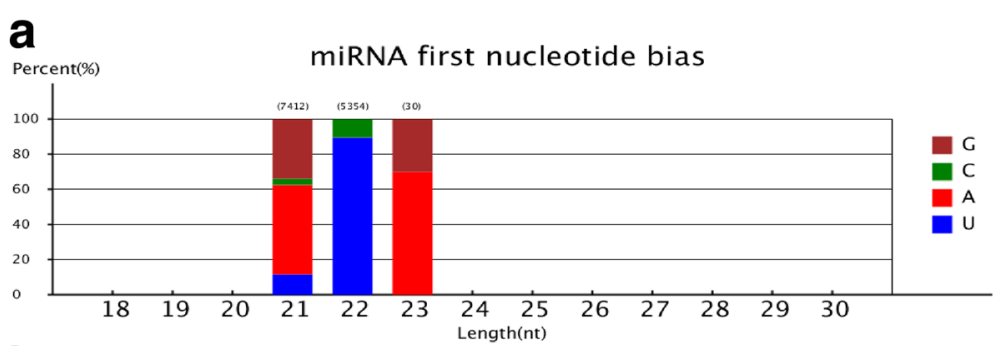

b
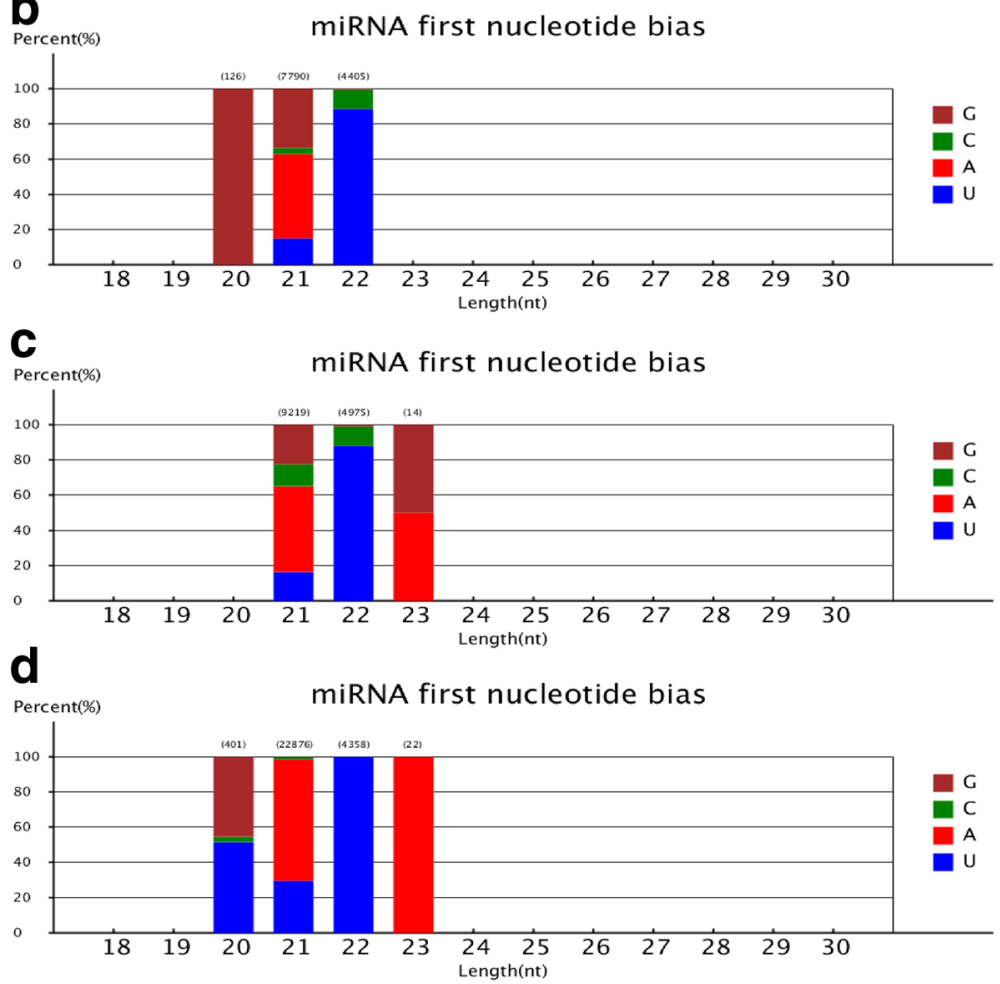

Fig. 4 First nucleotide bias of length classes of Camellia sinensis novel miRNAs under drought stress conditions. Drought stress conditions were as follows: a normal water supply, CK; b mild drought stress, T1; c moderate drought stress, T2; and $\mathbf{d}$ severe drought stress, T3. Brown, green, red, and blue correspond to guanine $(G)$, cytosine $(C)$, adenosine $(A)$, and uracil $(U)$, respectively

was found to be a significant determinant of drought stress tolerance in Brassica napus [45]. Some minerals have been shown to enhance the resistance of plants to drought stress [46]. On the basis of our results, we inferred that applications of D-alanine, sulfur, and minerals may help to alleviate drought stress in tea plants.

Sulfur metabolism and mineral absorption, the key enriched pathways along with D-alanine metabolism in CK vs. T1, CK vs. T2, and CK vs. T3, are illustrated in Fig. 8. Only one target gene of differentially expressed miRNAs was predicted: a gene encoding the $50 \mathrm{~S}$ ribosomal protein (GenBank accession number HP752910.1) targeted by miRNAs such as miR854, miR1858a, and miR530-5p. A previous study showed that the 50S ribosomal protein enhanced the drought resistance of barley by accelerating degradation of abnormal proteins under drought conditions [47]. In this study, we identified three genes related to the sulfur metabolism pathway that were targeted by drought-related miRNAs: serine acetyltransferase (EC 2.3.1.30) gene targeted by miR5563-5p and miR159a; ATP sulfurylase (EC 2.7.7.4) gene with seven members targeted by miR395a; and APS kinase (EC 2.7.1.25) gene with seven members targeted by miR395a. Sulfur has been shown to be a pivotal component in plant responses to abiotic stresses [48], suggesting that these targets of differentially expressed miRNAs play key roles in sulfur assimilation during drought stress in tea plants.

The five genes targeted by miR854 were associated with the mineral absorption pathway: one gene (DMT1) encoding a divalent metal-ion transporter-1 (AT1G47240.1), three CTR genes encoding copper transporters (KA299366.1, KA292949.1 and HP749962.1), and the ATOX1 gene (KA286605.1). 


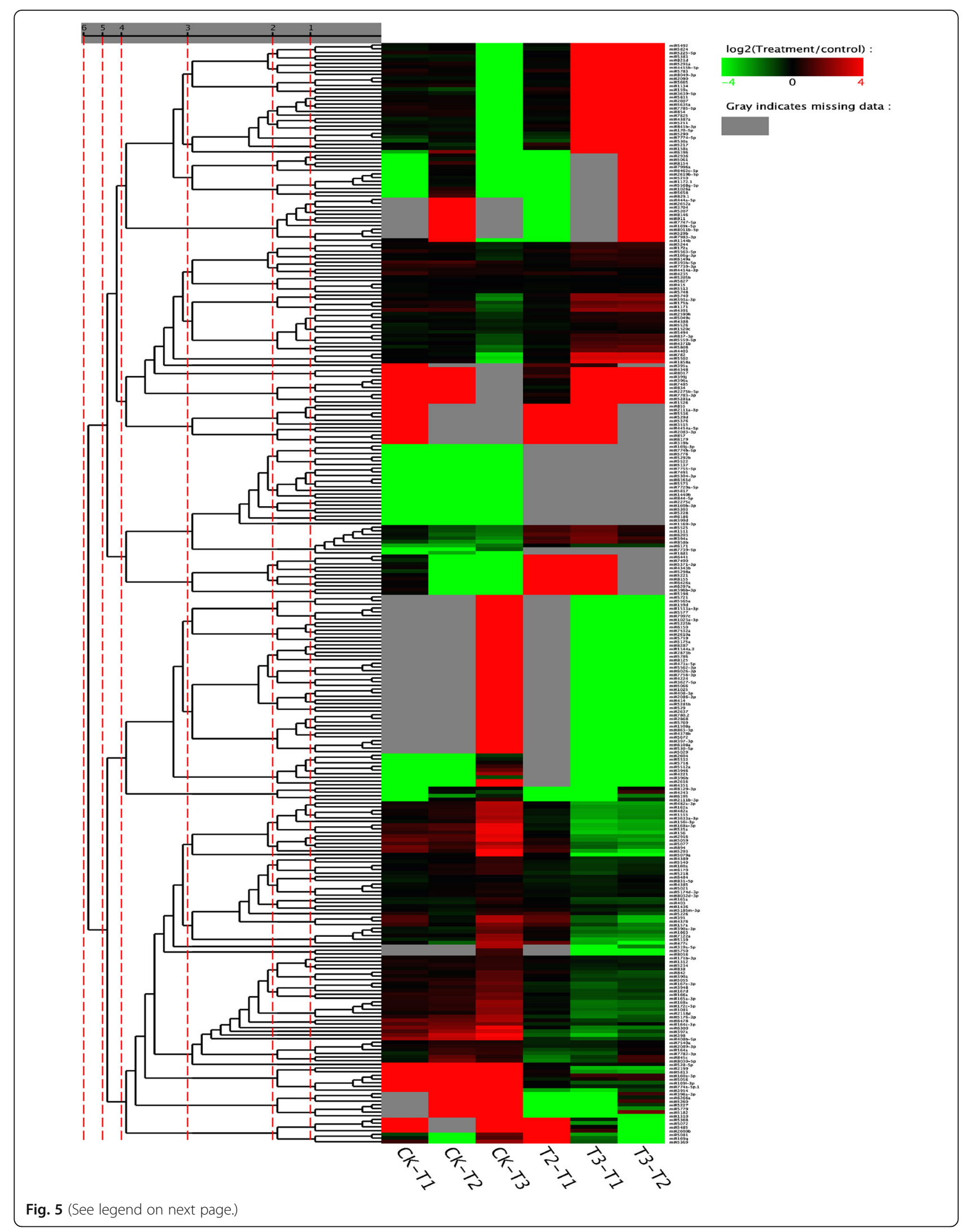


(See figure on previous page.)

Fig. 5 Heat map of Camellia sinensis conserved miRNAs differentially expressed between different drought stress conditions. Drought stress conditions were as follows: normal water supply (CK), mild drought stress (T1), moderate drought stress (T2), and severe drought stress (T3). Scale bar corresponds to miRNA relative expression levels; different hues indicate relative signal intensities. Red and green correspond respectively to up- or down-regulated expression of a given miRNA at each stage

DMT1 is highly relevant to mineral homeostasis [49] and CTR1 functions in maintaining copper homeostasis in different plant species [50]. ATOX1 is a copper metallochaperone protein. Therefore, copper homeostasis might be very important during the drought stress response in tea plants.

\section{Quantitative expression analysis of miRNAs during drought stress in $C$. sinensis}

The high-throughput sequencing results revealed differential expression of miRNAs under drought stress, and further analyses predicted the functions of the target genes. On the basis of those results, we selected 14 conserved miRNAs (csi-miR156, csi-miR159a, csi-miR165a3p, csi-miR854, csi-miR166a, csi-miR166g-3p, csimiR167d, csi-miR2199, csi-miR398, csi-miR408b-5p, csimiR435a, csi-miR6170, csi-miR894, and csi-miR395a) and seven novel miRNAs (csi-miR4, csi-miR7, csimiR12, csi-miR18, csi-miR24, csi-miR26, and csi-miR28), all possibly associated with the $C$. sinensis drought stress response, for validation of their expression profiles. In the $\mathrm{qPCR}$ analysis, expressions of all selected miRNAs were detected in the various treatments. According to their expression in $\mathrm{T} 1$ compared with that in $\mathrm{CK}$, the 21 miRNAs could be divided into two groups: group 1, which showed higher expression in T1 than in CK (Fig. 9a), and group 2, which showed lower expression in T1 than in CK (Fig. 9b).

Group 1 consisted of 13 miRNAs: csi-miR26, csimiR28, csi-miR12, csi-miR156, csi-miR159a, csi-miR398, csi-miR170, csi-miR4, csi-miR18, csi-miR165a-3p, csimiR408b-5p, csi-miR7, and csi-miR854 (Fig. 9a). Two miRNAs, namely csi-miR398 (conserved miRNA) and csi-miR7 (novel miRNA), were up-regulated during drought stress. The expression level of csi-miR398, which increased linearly in all samples, differed significantly among treatments. The expression level of csi-miR7 was significantly higher in T1 than in CK, but not significantly different among $\mathrm{T} 1, \mathrm{~T} 2$, and $\mathrm{T} 3$. The six conserved miRNAs (csi-miR156, csi-miR159a, csi-miR6170, csi-miR165a-3p, csi-miR408b-5p, and csimiR854) and five novel miRNAs (csi-miR26, csi-miR28, csi-miR12, csi-miR4, and csi-miR18) were up-regulated and then down-regulated in C. sinensis under drought stress. The expression levels of csi-miR165a-3p, csimiR159a, csi-miR408b-5p, csi-miR6170, and csi-miR854 were the highest in T1 and lowest in T3. The expression level of miR156 increased under drought stress, similar to the expression pattern of miR156 in A. thaliana under high salt and low temperature stresses [51]. The novel miRNAs csi-miR4, csi-miR18, csi-miR26, and csimiR28 showed the highest expression levels in T1 and the lowest expression levels in T3.

Group 2 contained eight miRNAs: the conserved miRNAs csi-miR166a, csi-miR166g-3p, csi-miR435a, csimiR2199, csi-miR894, csi-miR167d, and csi-miR395a; and the novel miRNA csi-miR24. These miRNAs were downregulated in C. sinensis during drought stress (Fig. 9b), implying that they were under negative regulation. The lowest expression levels of these miRNAs were in T3. Among these miRNAs, miR166 is a highly conserved drought-responsive miRNA [52-57] that is also known to be involved in root tip development. This implies that post-transcriptional regulation mediated by miR166 is a crucial regulatory pathway involved in plant root architecture and the drought response.

\section{Phase-specific miRNAs during drought stress in C. sinensis}

Based on our results, we constructed a diagram showing phase-specific miRNAs expressed under drought stress in C. sinensis (Fig. 10). Under normal water supply (CK), C. sinensis plants showed normal growth and development, and soil moisture content, leaf water content, electrical conductivity, and chlorophyll and MDA concentrations were all at normal levels. Seven miRNAs (miR166a, miR166g-3p, miR435a, miR894, miR2199, miR24, and miR395a) showed significantly higher expression levels in CK than in the T1, T2, and T3 treatments.

Under mild drought stress (T1), the leaves of $C$. sinensis plants showed slight curling and deformation; soil moisture, leaf water content, and chlorophyll (chlorophyll a, chlorophyll b and total chlorophyll) concentrations decreased, while leaf electrical conductivity and MDA concentration were barely affected. The 11 miRNAs (miR26, miR28, miR159a, miR6170, miR4, miR18, miR165a-3p, miR408b-5p, miR7, miR854, and miR167d) showed maximum expression levels in T1. The expression levels of miR435a and miR2199 were also relatively high in T1, compared with CK.

In $\mathrm{T} 2$, there was a further decrease in soil moisture content and the $C$. sinensis plants showed a loss of leaf luster, curling and wilting of tender leaves, decreased leaf water content and chlorophyll concentration, and slight increases in leaf electrical conductivity and MDA concentration. These changes reflected a declining photosynthetic electron transfer capability. The two miRNAs, miR12 and 


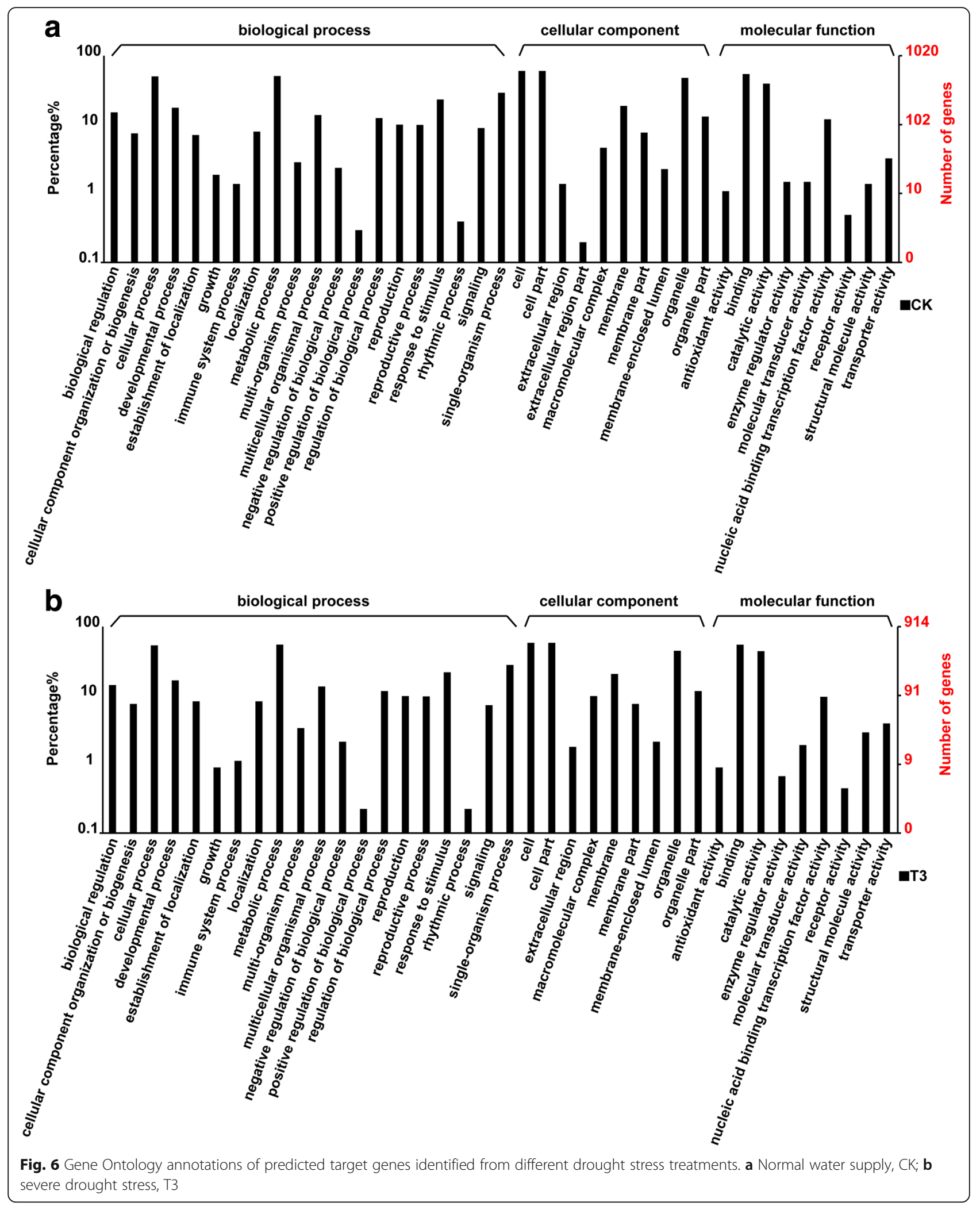




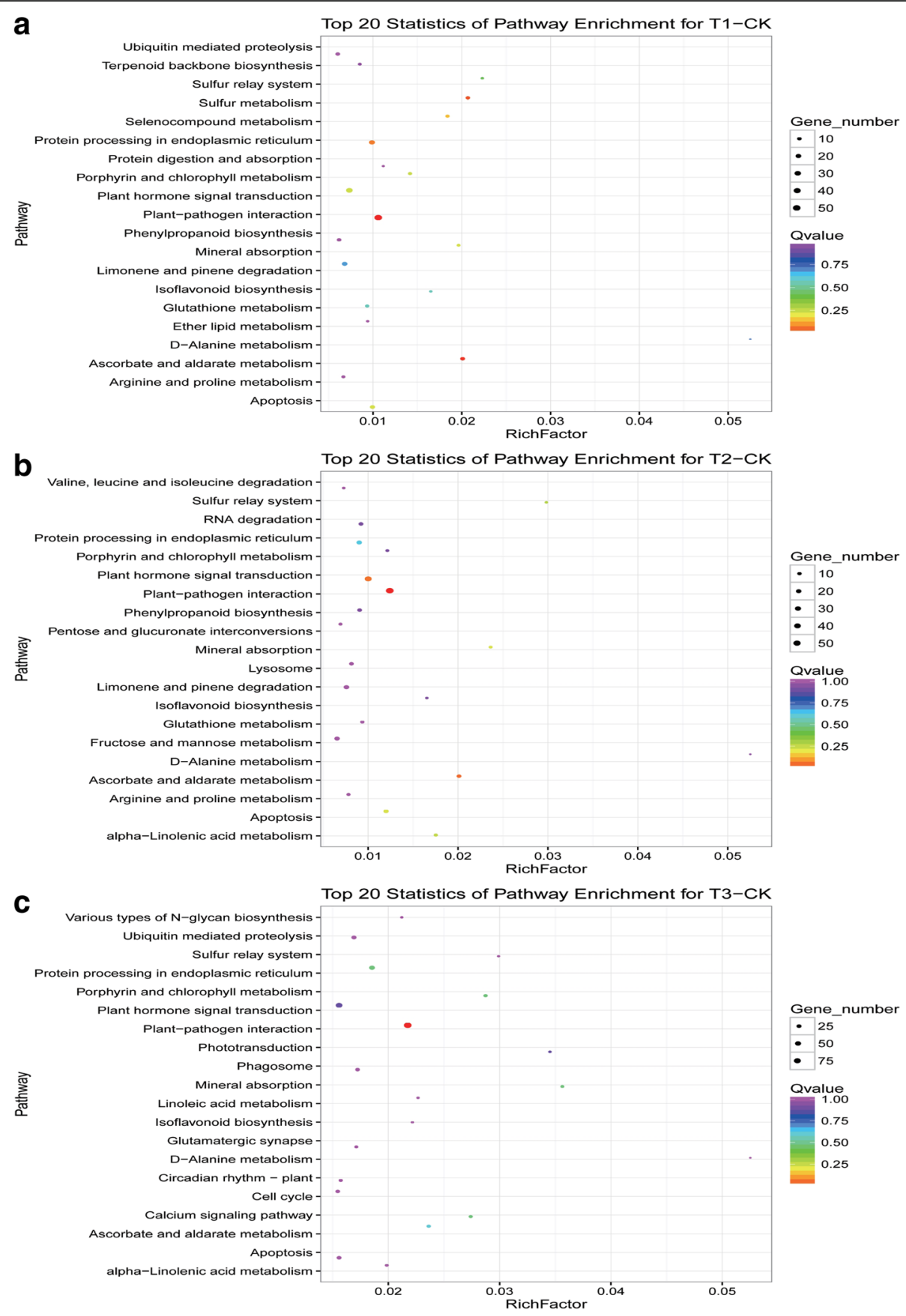

Fig. 7 Top 20 KEGG pathways enriched in conserved miRNA target genes differentially expressed between treatments. a Mild drought stress (T1) vs. normal water supply (CK); b moderate drought stress (T2) vs. CK; and c severe drought stress (T3) vs. CK. X-Axis indicates miRNA enrichment factors; $y$-axis corresponds to miRNA-enriched metabolic pathways

miR156, showed maximum expression levels in T2, and the expression levels of miR165a-3p, miR7, miR2199, and miR398 were also high.

In T3, leaves of C. sinensis plants withered completely and most branches died. Soil moisture, leaf water content, and leaf chlorophyll concentration continued to decline. In addition, the leaf MDA concentration and electrical conductivity markedly increased. The significant increase in cell electrolyte leakage was indicative of disruptions to plant cell structure and functional integrity, which ultimately affect plant growth and development. In T3, the only miRNA showing maximum expression was miR398, although there were also high expression levels of csi- 


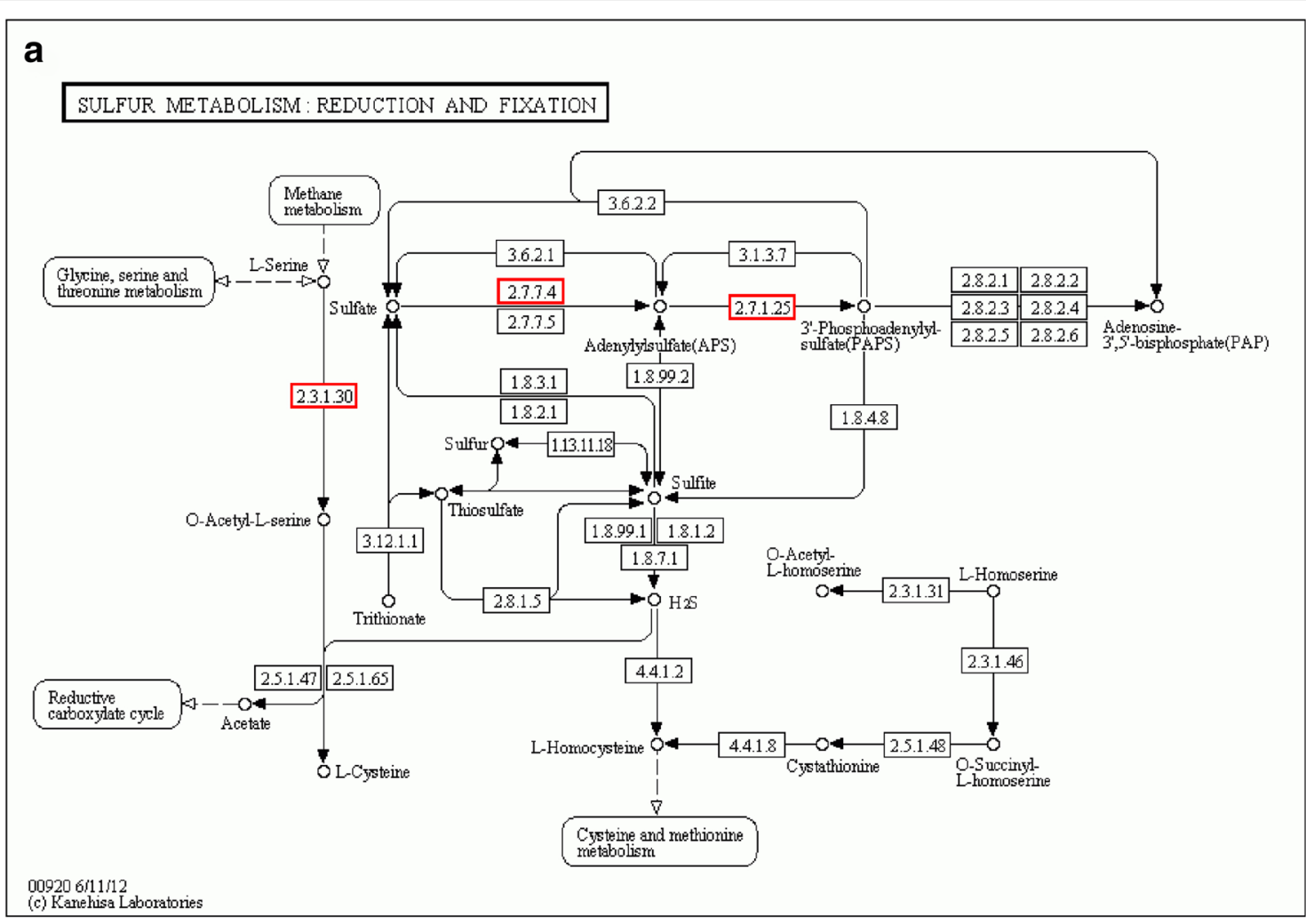

\section{b}

MINERAL ABSORPTION

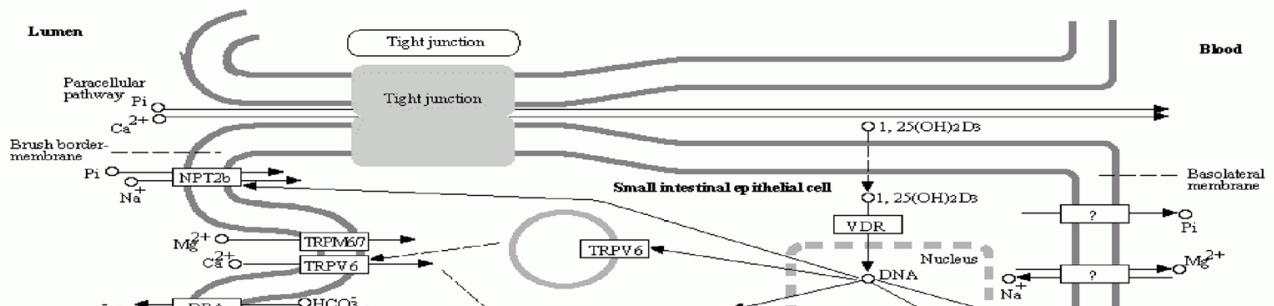

CiO $4 \mathrm{DRA}$

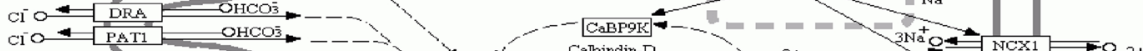

$\mathrm{PAT} 1=\mathrm{OHCO} 3$

CabP9K - -

$\mathrm{Cl}_{\mathrm{CLC} 2 \mathrm{O}} \mathrm{H} \mathrm{HO}_{3}^{-}$

Calbindim- $\mathrm{D}_{9 \mathrm{~K}}$

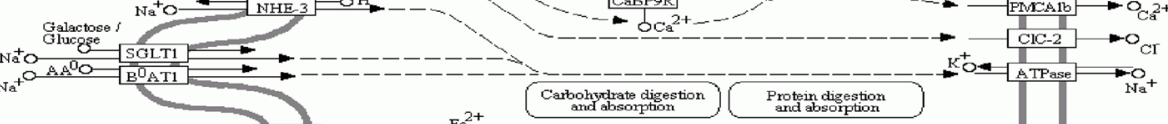

$\mathrm{Na}^{+} \mathrm{O}$
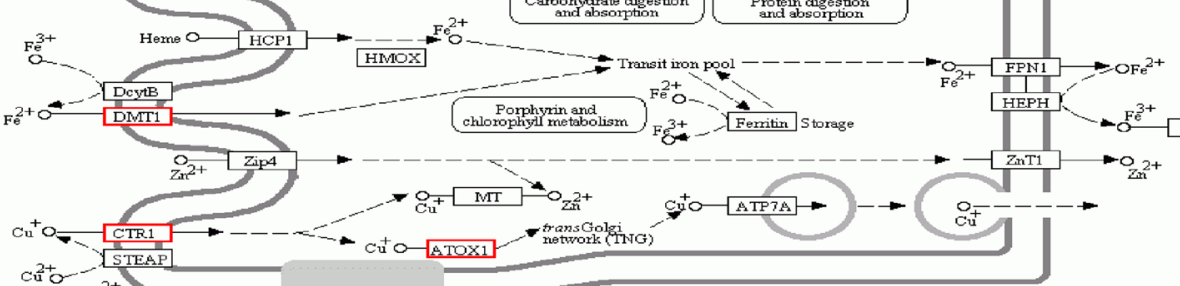

Paracellular

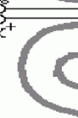

04978 3/31111

Fig. 8 (See legend on next page.) 
(See figure on previous page.)

Fig. 8 Targets of differentially expressed miRNAs in enriched pathways of sulfur metabolism and mineral absorption. a Schematic of sulfur metabolism pathway showing targets of miRNAs differentially expressed between normal water supply (CK) and mild (T1), moderate (T2), and severe (T3) drought conditions. b Schematic of mineral absorption pathway showing targets of miRNAs differentially expressed between normal water supply and mild, moderate, and severe drought conditions. Numbers in boxes are Enzyme Commission (EC) numbers. No map of D-alanine metabolism pathway and associated targets is shown

miR7 and miR2199. These results were consistent with those of the KEGG analysis, that metabolic pathways related to photosynthesis were significantly affected under T3. Since photosynthesis was not affected in the $\mathrm{T} 1$ and $\mathrm{T} 2$ treatments, these results suggested that photosynthesis in $C$. sinensis may not be significantly affected below a certain threshold of drought stress.

\section{Cloning and validation of miR166 target genes in C.} sinensis

To explore the regulatory functions of csi-miR166, we cloned the HD-Zip III subfamily transcription factors ATHB-14-like and ATHB-15-like, and then monitored their expression patterns under drought stress by qPCR. Both of these transcription factors were up-regulated under T1, T2, and T3 (Fig. 9c). Interestingly, the highest

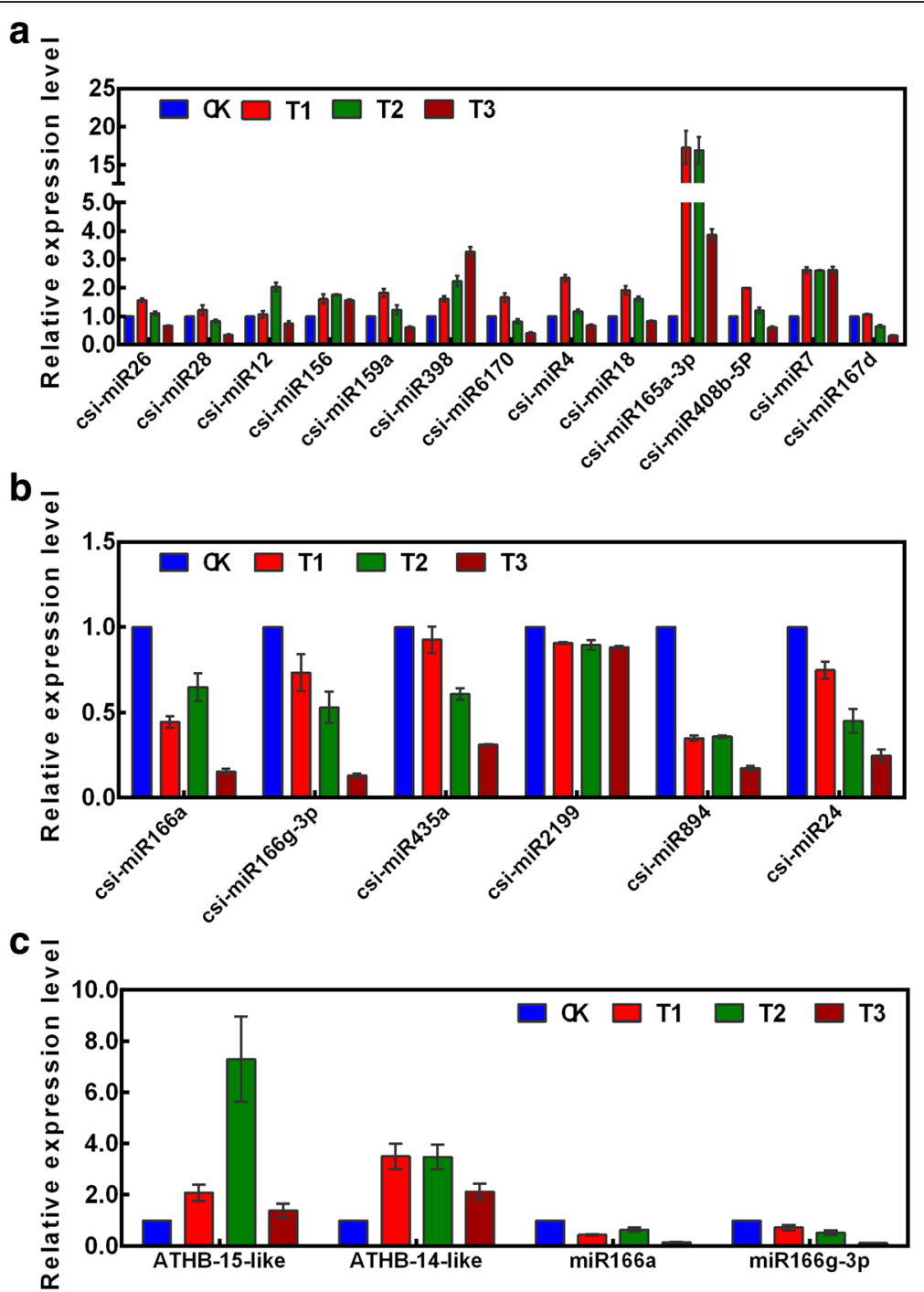

Fig. 9 Expression profiles of selected miRNAs and targets under different drought stress conditions. a-b Expression profiles of 21 selected conserved (A) and novel (B) miRNAs under normal water supply (CK), mild drought (T1), moderate drought (T2), and severe drought (T3) conditions. c Expression profiles of miR166s and their target genes (ATHB-14-like and ATHB-15-like) under CK, T1, T2, and T3 conditions. Microarray expression signals were obtained with three probe repeats. Error bars indicate SE based on three replicates 


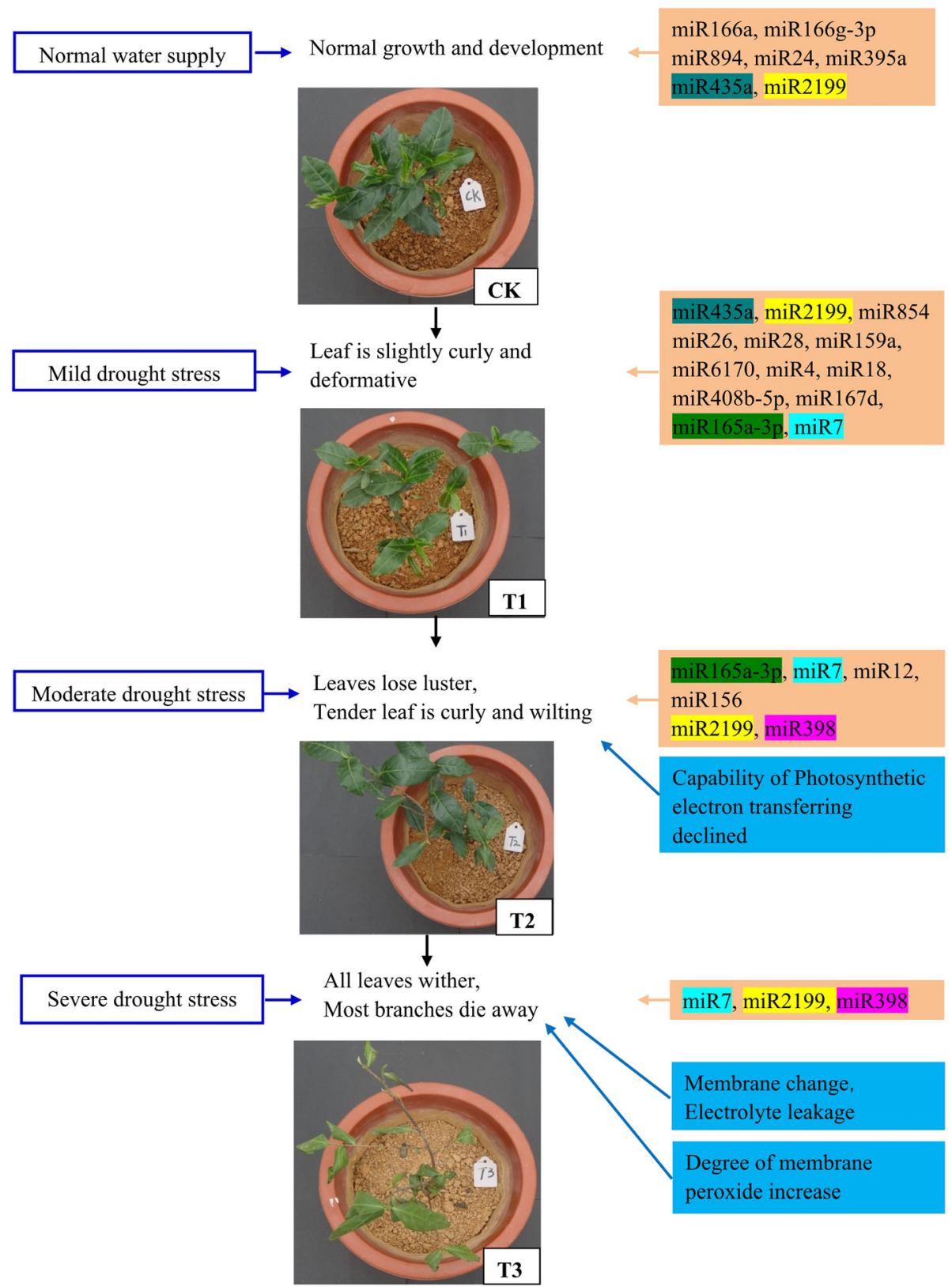

Fig. 10 Functional mechanisms underlying physiological changes and phase-specific miRNAs during drought stress in Camellia sinensis

expression level of $A T H B$-15-like was in T2, whereas $A T H B$-14-like was induced and maintained at higher levels under T1 and T2 than under T3. In contrast, miR166a and miR166g-3p were down-regulated under T1, T2, and T3, indicating that the expression levels of miR166s were negatively correlated with those of their target genes.

\section{Discussion}

Variation in sRNA length distribution and number of miRNAs in $C$. sinensis during drought stress

Analyses of $C$. sinensis sRNA length distributions in the absence of reference genome sequence calibrations revealed that the most common sRNA length in the four libraries was $24 \mathrm{nt}$, followed by $21 \mathrm{nt}$. These sRNA lengths, which varied during drought stress, are similar to those previously reported for plants such as $B$. napus [58] The 24-nt sRNAs accounted for 71.48\%, 65.87\%, $64.63 \%$, and $42.26 \%$ of total sRNAs in CK, T1, T2, and T3 samples, respectively. The proportion of 21-nt sRNAs was $11.50 \%$ (CK), $15.02 \%$ (T1), $15.57 \%$ (T2), and $33.25 \%$ (T3), indicating that an increasing number of 21nt sRNAs participate in biological regulation during drought stress in C. sinensis plants. Meanwhile, the proportion of miRNAs and the number and percentage of 
unique miRNAs tended to increase under drought stress (see data in Table 2). Thus, we inferred that Tieguanyin tea plants respond to drought stress by synthesizing and activating more miRNAs involved in drought regulation.

By using the genome of the model plant $A$. thaliana as a reference and comparing our sequence data with genetic data in the GenBank and Rfam databases, we annotated 883,059 (5.60\%), 970,915 (7.35\%), 1,110,918 (7.66\%), and 2,122,227 (15.78\%) miRNAs in the CK, T1, T2, and T3 libraries, respectively. Further comparison of miRNA sequencing data from the drought-treatment libraries with information in the $C$. sinensis transcriptome database led to the discovery of additional novel miRNAs and the precise locations of their target loci in the Tieguanyin tea plant genome. In total, 176 miRNA sequencing reads were identified and 4067 target loci were predicted.

Although many novel miRNAs were annotated in the four drought-stress libraries, some miRNAs remained unidentified, as has been the case in studies on other plants [59-61].

\section{Drought-related miRNAs in C. sinensis}

To elucidate the drought-resistance mechanisms $C$. sinensis, it is important to explore the spatio-temporal variations in miRNAs under drought stress. In this study, most miRNAs showed low abundance in droughtstressed C. sinensis, and only a few miRNA families were over-expressed, consistent with the results of other studies on plant miRNAs $[62,63]$. We also observed considerable differences in expression levels of miRNAs in the same family, such as miR166a and miR166g-3p. The expression patterns of miRNAs are highly conserved among different plants. For example, members of the miR156, miR166, and miR398 families are overexpressed in most plants [64-66]. Our results indicated that the expression patterns of these miRNAs in $C$. sinensis are similar to those in other plants. The results of this study revealed a relationship between stress resistance and conserved miRNAs such as miR156, miR166a, and miR398. This relationship has also been observed in other plants $[27,67,68]$, suggesting that these miRNAs might play key roles in the drought stress response in $C$. sinensis. The correlation between miRNAs and the drought stress response of $C$. sinensis requires further verification.

\section{Functions of genes targeted by drought-related miRNAs in C. sinensis}

Among the miRNAs identified in our study, miR398 plays a crucial regulatory role in respiration by targeting cytochrome c oxidase subunit $\mathrm{V}$, an enzyme involved in electron transport in the mitochondrial respiratory pathway $[69,70]$. Various studies have shown that miR398 is upregulated under drought stress in Triticum dicoccoides
[19], down-regulated in Zea mays [14], and both upregulated and down-regulated in Medicago truncatula $[16,20]$. In our study, miR398 was up-regulated in $C$. sinensis during drought stress, consistent with miR398 expression patterns observed in M. truncatula [16] and T. dicoccoides [19]. In a previous study, decreased miR398 expression levels coupled with increasing levels of $\mathrm{Cu} / \mathrm{Zn}$ superoxide dismutases $(\mathrm{Cu} / \mathrm{Zn}-\mathrm{SOD})$ in wild Ipomoea campanulata under drought stress confirmed the role of miR398 in drought stress tolerance. In another study, however, expression of miR398 did not correlate with $\mathrm{Cu} /$ $\mathrm{Zn}$-SOD accumulation in cultivated Jacquemontia pentantha [71]. These contrasting results indicate that the functions of miR398 differ among species, with the metabolic status of individual plants, and with the severity and duration of drought stress. Therefore, the regulatory mechanisms of miR398 in plants are still unknown.

One of the most highly conserved miRNAs, miR156, regulates the timing of developmental transitions [72]. Members of the miR156 family are known to be highly induced by heat stress and to play roles in heat stress memory in plants such as wheat (T. aestivum) [73], $B$. rapa [74], and $A$. thaliana [75]. Previous studies have found that csi-miR156 regulates $D F R$ transcription by controlling $S P L$ expression to influence catechin biosynthesis [76-78]. The accumulation of plant secondary metabolites is closely related to stress resistance in plants. We observed that miR156 was up-regulated under drought stress in C. sinensis, suggesting that this miRNA activates the catechin biosynthesis pathway in tea plants during drought stress. We inferred that miR156 enhances the resistance of tea plants to drought stress by inducing the accumulation of secondary metabolites.

Reyes et al. [79] reported that miR159 expression increased in germinating seeds of Arabidopsis treated with ABA and drought. Further studies showed that miR159a mediates the cleavage of MYB33 and MYB101 transcripts in A. thaliana $[79,80]$, while $M Y B$ transcription factors bind cis-elements in the promoter of the dehydration-responsive gene dehydration 22 (RD22) and activate $R D 22$ cooperatively. Osmotic stress tolerance in transgenic plants can be improved by over-expression of both $M Y C 2$ and MYB2 [81]. Microarray analyses have shown that miR167 is induced and up-regulated during drought stress in A. thaliana [51]. As a positive regulator of drought stress resistance, phospholipase D (PLD) is a predicted target of miR167. In $Z$. mays, the accumulation of PLD mRNA was shown to increase as the expression of miR167 decreased under drought stress [14]. Zhou et al. [18] reported that miR854 played a regulatory role in the drought stress response of rice, but its mechanism was unclear. In our study, the expression levels of miR167 and miR854 in C. sinensis increased 
under mild drought stress, but decreased with increasing severity of drought stress (Fig. 9a and b). Thus, we hypothesize that mild drought stress up-regulates miR167 and miR854.

In our study, csi-miR166a, csi-miR166g-3p, csimiR435a, csi-miR894, csi-miR2199, csi-miR24, and csimiR395a were down-regulated in C. sinensis during drought stress. The expression pattern of miR166 in response to drought stress differs among plant species; it is down-regulated in barley [15] and T. dicoccoides [19] but up-regulated in M. truncatula, especially in roots [16]. According to other reports, miR166a overexpression reduces the number of lateral roots in $M$. truncatula [82] and strengthens vascular development and enlarge shoot apical meristems in A. thaliana [83]. A previous study indicated that limitation of external water supply changes the architecture of the plant root system to improve water absorption efficiency [84]. In the present study, the miR166 family members csimiR166a and csi-miR166g-3p were found to negatively regulate their target genes in $C$. sinensis during drought stress. This pattern of regulation is consistent with those observed in cotton under salt stress [63] and in wheat under drought stress shock [19]. The co-adjustment of the miR166 family and its targets $A T H B$-14-like and $A T H B$-15-like indicate the existence of negative feedback regulation in the miRNA pathway. We observed the strongest up-regulation of $A T H B$-15-like under moderate drought stress, indicating that the miR166 family strongly regulated its expression at this stage. This result implies that the expression of target genes of miR166 family members is regulated by dynamic changes in expression levels of their corresponding miRNAs. To adapt to environmental changes, tea plants can correspondingly self-regulate in response to different levels of drought stress. The predicted target of miR395, a miRNA enriched under drought treatment, is the sulfate transporter SULTR2 [85]. In regard to sulfate transporters, miR395 plays an important role in reequilibrating the sulfate flux in different tissues and improving drought stress resistance in plants [86, 87]. ATP sulfurylase has a crucial function in the formation of APS from ATP and sulfate $[88,89]$, a process catalyzed by APS kinase (EC 2.7.1.25; 7 unigenes) [90]. In our study, ATP sulfurylase and $A P K$ were up-regulated in tea plants under drought stress, and their encoding genes were predicted targets of miR395a.

\section{D-alanine metabolism, sulfur metabolism, and mineral absorption metabolism play important roles in the drought stress response of $C$. sinensis}

Analyses of the target genes of miRNAs related to drought stress indicated that D-alanine metabolism, sulfur metabolism, and mineral absorption metabolism were highly enriched pathways. Thus, these pathways might play important roles in the drought stress response of C. sinensis. In some species, these pathways are involved the responses to various abiotic stresses. For example, the formation of free amino acids in cotton under high temperature stress may play a significant role in maintaining cell water potential, eliminating toxicity, and storing nitrogen [91]; sulfur metabolism was found to be an important component of the cold resistance mechanism of cassava [92]; in B. napus [45], sulfur uptake affected the availability of total sulfur, which played important roles in alleviating damage caused by drought stress. Some minerals have been shown to enhance the drought tolerance of plants by increasing the concentration of antioxidants [46]. Therefore, D-alanine metabolism, sulfur metabolism, and mineral absorption pathways are likely to play important roles in the drought stress response of C. sinensis. Interestingly, a role of D-alanine metabolism in plant abiotic stress responses has seldom been reported, and warrants further research.

Under drought stress, a series of physiological, biochemical, and molecular changes lead to adaptive drought responses in plants [93, 94]. Previous studies have found that drought stress affects carbon dioxide assimilation rates and photosynthetic pigment synthesis $[93,95]$ and results in the generation of reactive oxygen species that cause oxidative damage as measured by lipid peroxidation [96]. We observed that the expression of miRNAs in C. sinensis differed significantly among drought treatments with different degrees of severity. Some specific metabolic pathways activated under drought stress in tea plants were identified. These findings provide new clues about the molecular mechanism of the drought stress response in tea plants.

\section{Conclusions}

We analyzed drought-responsive miRNAs in tea plants, and found that most of their targets were related to transcriptional regulation. The most highly enriched pathways under drought stress were Dalanine metabolism, sulfur metabolism, and mineral absorption metabolism. Based on the results of qPCR analyses, 21 miRNAs associated with the drought stress response were divided into two groups. The results of this study showed that the expressions of phase-specific miRNAs vary with morphological, physiological and biochemical changes that occur under drought stress. These findings will be useful for research on drought resistance and provide insights into the mechanisms of drought adaptation and resistance in C. sinensis. 


\section{Additional files}

Additional file 1: Table S1. Primers used for $q P C R$ amplification of Camellia sinensis 'Tieguanyin' miRNAs. (DOCX $14 \mathrm{~kb}$ )

Additional file 2: Table S2. Summary of novel miRNA targets predicted from Tieguanyin RNA sequences generated under different drought stress conditions. (DOCX $13 \mathrm{~kb}$ )

Additional file 3: Figure S1. Heat map of Camellia sinensis novel miRNAs differentially expressed between different drought stress conditions (CK, normal water supply; T1, mild drought stress; T2, moderate drought stress; T3, severe drought stress). (JPEG $1290 \mathrm{~kb}$ )

Additional file 4: Table S3. Camellia sinensis known miRNAs differentially expressed between normal water supply and mild drought stress (CK vs. T1). (XLSX 14 kb)

Additional file 5: Table S4. Camellia sinensis known miRNAs differentially expressed between normal water supply and moderate drought stress (CK vs. T2). (XLSX 15 kb)

Additional file 6: Table S5. Camellia sinensis known miRNAs differentially expressed between normal water supply and severe drought stress (CK vs. T3). (XLSX $21 \mathrm{~kb})$

Additional file 7: Figure S2. Gene Ontology annotations of predicted target genes of miRNAs identified from mild drought stress treatment (T1). (JPEG 2589 kb)

Additional file 8: Figure S3. Gene Ontology annotations of predicted target genes of miRNAs identified from moderate drought stress treatment (T2). (JPEG $2596 \mathrm{~kb}$ )

Additional file 9: Table S6. GO statistics of Camellia sinensis known miRNAs during drought stress. (XLS $32 \mathrm{~kb}$ )

Additional file 10: Table S7. KEGG pathways enriched in Camellia sinensis known miRNAs during drought stress. (XLS 984 kb)

Additional file 11: Table S8. Top 20 KEGG pathways enriched in Camellia sinensis known miRNAs during drought stress. (XLS $167 \mathrm{~kb}$ )

Additional file 12: Figure S4. Statistical summary of KEGG enriched pathways of top 20 target genes of novel miRNAs. (JPEG $5124 \mathrm{~kb}$ )

\section{Abbreviations}

ABA: Abscisic acid; cDNA: Complementary DNA; DNA: Deoxyribonuclease acid; h: Hour; MDA: Malondialdehyde; MFE: Minimum free energy; miRNA: microRNA; mRNA: messenger RNA; PCR: Polymerase chain reaction; qPCR: Real time quantitative PCR; RNA: Ribonuclease acid; rRNA: Ribonuclease RNA; s: Second; scRNA: Small cytosol RNA; snoRNA: Small nucleolar RNA; snRNA: Small nuclear RNA; tRNA: Transfer RNA

\section{Acknowledgements}

The authors wish to thank Zihao Zhang for technical assistance in sample treatment.

\section{Funding}

The fees for high-throughput sequencing and data processing were supported by the Major Science and Technology Project in Fujian Province (2015NZ0002-1) and the Project of Department Education of Fujian Province (JA15143); the fees for real-time quantitative PCR detection were supported by the National Natural Science Foundation of China (31600555) and the Natural Science Foundation of Fujian Province (12171082); the publication fees were supported by the Construction of High-Level University Program of Fujian Agriculture and Forestry University (612014007). The funding body did not play a role in the design of the study and collection, analysis, and interpretation of data and in writing the manuscript.

\section{Availability of data and materials}

All data presented in this study are provided either in the manuscript or additional files.

\section{Authors' contributions}

$Z L, Y G$, and SZ designed the work; YG, SZ, and XC performed the experiments and wrote the paper; $Z \mathrm{~L}, \mathrm{YG}, \mathrm{SZ}, \mathrm{ZW}$, and $\mathrm{YL}$ analyzed the data;
$Z L, C Z, X C$, and $C Y$ helped to perform the sequence analysis and revised the paper carefully. All authors have read and approved the manuscript.

\section{Ethics approval and consent to participate}

The tea cultivar 'Tieguanyin' used in this study was planted in College of Horticulture, Fujian Agriculture and Forestry University, Fuzhou, Fujian, China. No specific permits were required for plant collection. The study did not require ethical approval or consent as no endangered or protected plant species were involved.

\section{Consent for publication}

Not applicable.

\section{Competing interests}

The authors declare that they have no competing interests.

\section{Publisher's note}

Springer Nature remains neutral with regard to jurisdictional claims in published maps and institutional affiliations.

Received: 16 May 2017 Accepted: 10 November 2017

Published online: 21 November 2017

\section{References}

1. Cheruiyot EK, Mumera LM, Ngetich WK, Hassanali A, Wachira FN. High fertilizer rates increase susceptibility of tea to water stress. J Plant Nutr. 2009:33(1):115-29.

2. Liu SC, Yao MZ, Ma CL, Jin JQ, Ma JQ, Li CF, Chen L. Physiological changes and differential gene expression of tea plant under dehydration and rehydration conditions. Scientia Horticulture. 2015;184:129-41.

3. Das A, Mukhopadhyay M, Sarkar B, Saha D, Mondal TK. Influence of drought stress on cellular ultrastructure and antioxidant system in tea cultivars with different drought sensitivities. J Environ Biol. 2015:36(4):875-82.

4. Maritim TK, Kamunya SM, Mireji P, Wendia CM, Muoki RC, Cheruiyot EK, Wachira FN. Physiological and biochemical response of tea [Camellia sinensis (L.) O. Kuntze] to water-deficit stress. J Horticultural Sci Biotechnol. 2015; 90(4):395-400

5. Gupta S, Bharalee R, Bhorali P, Bandyopadhyay T, Gohain B, Agarwal N, Ahmed P, Saikia H, Borchetia S, Kalita MC, Handique AK, Das S. Identification of drought tolerant progenies in tea by gene expression analysis. Funct Integr Genomics. 2012;12(3):543-63.

6. Gupta S, Bharalee R, Bhorali P, Das SK, Bhagawati P, Bandyopadhyay T, Gohain B, Agarwal N, Ahmed P, Borchetia S, Kalita MC, Handique AK, Das S. Molecular analysis of drought tolerance in tea by cDNA-AFLP based transcript profiling. Mol Biotechnol. 2013;53(3):237-48.

7. Das A, Das S, Mondal TK. Identification of differentially expressed gene profiles in young roots of tea [Camellia sinensis (L.) O. Kuntze] subjected to drought stress using suppression subtractive hybridization. Plant Mol Biol Report. 2012;30(5):1088-101.

8. Liu SC, Jin JQ, Ma JQ, Yao MZ, Ma CL, Li CF, Ding ZT, Chen L. Transcriptomic analysis of tea plant responding to drought stress and recovery. PLoS One. 2016;11(1):e0147306.

9. Wang WD, Xin $\mathrm{HH}$, Wang ML, Ma OP, Wang L, Kaleri NA, Wang YH, Li XH. Transcriptomic analysis reveals the molecular mechanisms of droughtstress-induced decreases in Camellia sinensis leaf quality. Front Plant Sci. 2016;7(795):385

10. Shriram V, Kumar V, Devarumath RM, Khare TS, Wani SH. MicroRNAs as potential targets for Abiotic stress tolerance in plants. Front Plant Sci. 2016; 7(235):817.

11. Zhang B. MicroRNA: a new target for improving plant tolerance to abiotic stress. J Exp Bot. 2015;66(7):1749.

12. Barrera-Figueroa BE, Wu Z, Liu R. Abiotic stress-associated microRNAs in plants: discovery, expression analysis, and evolution. Front Biol. 2013;8(2):189-97.

13. Li WX, Oono Y, Zhu J, He XJ, JM W, lida K. The Arabidopsis NFYA5 transcription factor is regulated transcriptionally and posttranscriptionally to promote drought resistance. Plant Cell. 2008;20(8):2238-51.

14. Wei LY, Zhang DF, Xiang F, Zhang ZX. Differentially expressed miRNAs potentially involved in the regulation of defense mechanism to drought stress in maize seedlings. Int J Plant Sci. 2009;170(8):979-89. 
15. Kantar M, Unver T, Budak H. Regulation of barley miRNAs upon dehydration stress correlated with target gene expression. Funct Integr Genomics. 2010; 10(4):493-507

16. Trindade I, Capitao C, Dalmay T, Fevereiro M, Santos D. miR398 and miR408 are up-regulated in response to water deficit in Medicago truncatula. Planta. 2010;231(3):705-16.

17. Chen X, Yang RF, Li WC, FL F. Identification of 21 microRNAs in maize and their differential expression under drought stress. Afr J Biotechnol. 2010;9(30):4741-53.

18. Zhou L, Liu Y, Liu Z, Kong D, Duan M, Luo L. Genome-wide identification and analysis of drought-responsive microRNAs in Oryza sativa. J Exp Bot. 2010;61(15):4157-68

19. Kantar M, Lucas SJ, Budak H. MiRNA expression patterns of Triticum dicoccoides in response to shock drought stress. Planta. 2011;233(3):471-84.

20. Wang T, Chen L, Zhao M, Tian Q, Zhang WH. Identification of droughtresponsive microRNAs in Medicago truncatula by genome-wide highthroughput sequencing. BMC Genomics. 2011;12(1):367.

21. Zhang X, Zou Z, Gong P, Zhang J, Ziaf K, Li H, Xiao F, Ye Z. Over-expression of microRNA169 confers enhanced drought tolerance to tomato. Biotechnol Lett. 2011;33(2):403-9.

22. Eldem $\mathrm{V}$, Okay $\mathrm{S}$, Unver T. Plant micrornas: new players in functional genomics. Turk J Agric For. 2012;37(1):1-21.

23. Gupta OP, Meena NL, Sharma I, Sharma P. Differential regulation of microRNAs in response to osmotic, salt and cold stresses in wheat. Mol Biol Rep. 2014;41(7):4623-9.

24. Pandey R, Joshi G, Bhardwaj AR, Agarwal M, Katiyar-Agarwal S. A comprehensive genome-wide study on tissue-specific and abiotic stressspecific miRNAs in Triticum aestivum. PLoS One. 2014;9(4):e95800.

25. Hajyzadeh M, Turktas M, Khawar KM, Unver T. miR408 over expression causes increased drought tolerance in chickpea. Gene. 2015;555(2):186-93.

26. Fan GQ, Lu Y, Deng MJ, Niu SY, Zhao ZL, Dong YP, Li YS. Comparative analysis of microRNAs and putative target genes in hybrid clone Paulownia 'yuza 1' under drought stress. Acta Physiol Plant. 2016;38(8):203.

27. Ding YF, Tao YL, Zhu C. Emerging roles of microRNAs in the mediation of drought stress response in plants. J Exp Bot. 2013:64(11):3077-86.

28. Das A, Mondal TK. Computational identification of conserved microRNAs and their targets in tea (Camellia sinensis). Am J Plant Sci. 2010;1(2):77-86.

29. Mohanpuria P, Yadav SK. Characterization of novel small RNAs from tea (Camellia sinensis L.). Mol Biol Rep. 2012;39(4):3977-86.

30. Zhang Y, Zhu X, Chen X, Song C, Zou Z, Wang Y, Wang M, Fang W, Li X. Identification and characterization of cold-responsive microRNAs in tea plant (Camellia sinensis) and their targets using high-throughput sequencing and degradome analysis. BMC Plant Biol. 2014;14(1):271.

31. Liu SC, YX X, Ma JQ, Wang WW, Chen W, Huang DJ, Fang J, Li XJ, Chen L. Small RNA and degradome profiling reveals important roles for microRNAs and their targets in tea plant response to drought stress. Physiol Plant. 2016; 158(4):435-51.

32. Upadhyaya H, Panda SK, Dutta BK. Variation of physiological and antioxidative responses in tea cultivars subjected to elevated water stress followed by rehydration recovery. Acta Physiol Plant. 2008;30(4):457-68.

33. Amsellem L, McKey DB. Integrating phenological, chemical and biotic defences in ant-plant protection mutualisms: a case study of two myrmecophyte lineages. Chemoecology. 2006;16(4):223-34.

34. Xing Y, Li X, Xu Q, Jiang YJ, Li W. Effects of chitosan-based coating and modified atmosphere packaging (MAP) on browning and shelf life of fresh-cut lotus root (Nelumbo nucifera Gaerth). Innovative Food Sci Emerg Technol. 2010;11(4):684-9.

35. Luo X, Huang Q. Studies on the cold resistance of cassava. J Agric Sci. 2012; 4(6):104-19.

36. Meyers BC, Axtell MJ, Bartel B, Bartel DP, Baulcombe D, Bowma JL, Cao XF, Carrington JC, Chen XM, Green PJ, Griffiths-Jonesj S, Jacobsenk SE, Mallory AC, Martienssen RA, Poethign RS, Qi YJ, Vaucheret H, Voinnet O, Watanabe Y, Weigel D, Zhu JK. Criteria for annotation of plant MicroRNAs. Plant Cell. 2008:20(12):3186-90.

37. Allen E, Xie Z, Gustafson AM, Carrington JC. MicroRNA-directed phasing during trans-acting siRNA biogenesis in plants. Cell. 2005;121(2):207-21.

38. Schwab R, Palatnik JF, Riester M, Schommer C, Schmid M, Weigel D. Specific effects of microRNAs on the plant transcriptome. Dev Cell. 2005:8(4):517-27.

39. Schmittgen TD, Livak KJ. Analyzing real-time PCR data by the comparative CT method. Nat Protoc. 2008;3(6):1101-8.

40. Kozomara A. Griffiths-Jones S: miRBase: annotating high confidence microRNAs using deep sequencing data. Nucleic Acids Res. 2014; 42(D1):68-73.
41. Nawrocki EP, Burge SW, Bateman A, Daub J, Eberhardt RY, Eddy SR, Floden EW, Gardner PP, Jones TA. John Tate, Finn RD: Rfam 12.0: updates to the RNA families database. Nucleic Acids Res. 2015;43(D1):130-37.

42. Czech B, Hannon GJ. Small RNA sorting: matchmaking for Argonautes. Nat Rev Genet. 2011;12(1):19-31.

43. Kim D, Langmead B, Salzberg SL. HISAT: a fast spliced aligner with low memory requirements. Nat Methods. 2015;12(4):357-60.

44. Liang WS. Drought stress increases both cyanogenesis and $\beta$-cyanoalanine synthase activity in tobacco. Plant Sci. 2003;165(5):1109-15.

45. Bok-Rye L, Rashed Z, Jean-Christophe A, Alain O, Tae-Hwan K. Sulfur use efficiency is a significant determinant of drought stress tolerance in relation to photosynthetic activity in Brassica napus cultivars. Front Plant Sci. 2016:7(704):459.

46. Waraich EA, Ahmad R, Ashraf MY. Role of mineral nutrition in alleviation of drought stress in plants. Aust J Crop Sci. 2011:5(6):764-77.

47. Ghabooli M, Khatabi B, Ahmadi FS, Sepehri S, Mirzaei M, Amirkhani A, JorrínNovo J, Salekdeh GH. Proteomics study reveals the molecular mechanisms underlying water stress tolerance induced by Piriformospora indica in barley. J Proteome. 2013:94(20):289-301.

48. Chan KX, Wirtz M, Phua SY, Estavillo GM, Pogson BJ. Balancing metabolites in drought: the sulfur assimilation conundrum. Trends Plant Sci. 2013;18(1):18-29.

49. Kaiser BN, Moreau S, Castelli J, Thomson R, Lambert A, Bogliolo S, Puppo A, Day DA. The soybean NRAMP homologue, GmDMT1, is a symbiotic divalent metal transporter capable of ferrous iron transport. PLANT J. 2003;35(3):295-304.

50. Meng $Y, \mathrm{Li} X \mathrm{XH}$, Xiao JH, Wang SP. Molecular and functional analyses of COPT/Ctr-type copper transporter-like gene family in rice. BMC Plant Biol. 2011;11(1):69

51. Liu HH, Tian X, Li YJ, CA W, Zheng CC. Microarray-based analysis of stressregulated microRNAs in Arabidopsis thaliana. RNA. 2008;14(5):836-43.

52. Aravind J, Rinku S, Pooja B, Shikha M, Kaliyugam S, Mallikarjuna MG, Kumar A, Rao AR, Nepolean T. Identification, characterization, and functional validation of drought-responsive microRNAs in subtropical maize inbreds. Front Plant Sci. 2017:8:941

53. Chen Q, Li M, Zhang Z, Tie W, Chen X, Jin L, Zhai N, Zheng Q, Zhang J, Wang $R, X u G$, Zhang $H$, Liu P, Zhou H. Integrated mRNA and microRNA analysis identifies genes and small miRNA molecules associated with transcriptional and post-transcriptional-level responses to both drought stress and re-watering treatment in tobacco. BMC Genomics. 2017;18(1):62.

54. Akdogan G, Tufekci ED, Uranbey S, Unver T. miRNA-based drought regulation in wheat. Funct Integr Genomics. 2016;16(3):221.

55. Ágyi Á, Havelda Z. Analysis of gradient-like expression of miR167 in Arabidopsis thaliana embryonic tissue. J Plant Biol. 2013;56(5):336-44.

56. Zhu H, Hu F, Wang R, Zhou X, Sze SH, Liou LW, et al. Arabidopsis Argonaute 10 specifically sequesters miR166/165 to regulate shoot apical meristem development. Cell. 2011;145(2):242-56.

57. Jung JH, Park CM. MIR166/165 genes exhibit dynamic expression patterns in regulating shoot apical meristem and floral development in Arabidopsis. Planta. 2007:225(6):1327-38.

58. Zhao Y, Wang M, Fu S, Yang W, Qi C, Wang X, Small RNA. Profiling in two Brassica napus cultivars identifies microRNAs with oil production-and development-correlated expression and new small RNA classes. Plant Physiol. 2012;158(2):813-23.

59. Chao YT, CL S, Jean WH, Chen WC, Chang YCA, Shih MC. Identification and characterization of the microRNA transcriptome of a moth orchid Phalaenopsis aphrodite. Plant Mol Biol. 2014;84(4-5):529-48.

60. Li J, LQ W, Zheng WY, Wang RF, Yang LX. Genome-wide identification of microRNAs responsive to high temperature in rice (Oryza sativa) by highthroughput deep sequencing. J Agron Crop Sci. 2014;201(5):379-88.

61. Khaldun ABM, Huang W, Liao S, LV H, Wang Y. Identification of microRNAs and target genes in the fruit and shoot tip of Lycium chinense: a traditional Chinese medicinal plant. PLoS One. 2015;10(1):e0116334.

62. Zhao CZ, Xia H, Frazier TP, Yao YY, Bi YP, Li AQ, Li MJ, Li CS, Zhang BH, Wang $X J$. Deep sequencing identifies novel and conserved microRNAs in peanuts (Arachis hypogaea L.). BMC Plant Biol. 2010;10(1):3.

63. Xie F, Wang $Q$, Sun $R$, Zhang B. Deep sequencing reveals important roles of microRNAs in response to drought and salinity stress in cotton. J Exp Bot. 2015:66(3):789-804

64. Burklew CE, Ashlock J, Winfrey WB, Zhang BH. Effects of aluminum oxide nanoparticles on the growth, development, and microRNA expression of tobacco (Nicotiana tabacum). PLoS One. 2012;7(5):e34783. 
65. Devi SJSR, Madhav MS, Kumar GR, Goel AK, Umakanth B, Jahnavi B, Viraktamath BC. Identification of abiotic stress miRNA transcription factor binding motifs (TFBMs) in rice. Gene. 2013;531(1):15-22.

66. Wang B, Sun $Y$, Song N, Wei JP, Wang XJ, Feng H, Yin ZY, Kang ZS. MicroRNAs involving in cold, wounding and salt stresses in Triticum aestivum L. Plant Physiol Biochem. 2014;80:90-6.

67. Iyer NJ, Jia X, Sunkar R, Tang G, Mahalingam R. MicroRNAs responsive to ozone-induced oxidative stress in Arabidopsis thaliana. Plant Signal Behav. 2012;7(4):484-91.

68. Lin YL, Lai ZX. Comparative analysis reveals dynamic changes in miRNAs and their targets and expression during somatic embryogenesis in longan (Dimocarpus longan lour.). PLoS One. 2013;8(4):e60337.

69. Jones-Rhoades MW, Bartel DP. Computational identification of plant microRNAs and their targets, including a stress induced miRNA. Mol Cell. 2004;14(6):787-99.

70. Sunkar R, Zhu JK. Novel and stress-regulated microRNAs and other small RNAs from Arabidopsis. Plant Cell. 2004;16(8):2001-19.

71. Ghorecha V, Patel K, Ingle S, Sunkar R, Krishnayya NSR. Analysis of biochemical variations and microRNA expression in wild (Ipomoed campanulata) and cultivated (Jacquemontia pentantha) species exposed to in vivo water stress. Physiol Mol Biol Plants. 2014:20(1):57-67.

72. Cho SH, Coruh C, Axtell MJ. miR156 and miR390 regulate tasiRNA accumulation and developmental timing in Physcomitrella patens. Plant Cell. 2012;24(12):4837-49.

73. Xin M, Wang Y, Yao Y, Xie C, Peng H, Ni Z, et al. Diverse set of microRNAs are responsive to powdery mildew infection and heat stress in wheat (Triticum aestivum L.). BMC Plant Biol. 2010;10(1):123.

74. Yu X, Wang H, Lu Y, Ruiter M, Cariaso M, Prins M, Tunen A, He YK. Identification of conserved and novel microRNAs that are responsive to heat stress in Brassica rapa. J Exp Bot. 2012;63(2):1025-38.

75. Stief A, Altmann S, Hoffmann K, Pant BD, Scheible WR, Bäurle I. Arabidopsis miR156 regulates tolerance to recurring environmental stress through SPL transcription factors. Plant Cell. 2014;26(4):1792-807.

76. Sun P, Cheng C, Lin Y, Zhu Q, Lin J, Lai Z. Combined small RNA and degradome sequencing reveals complex microRNA regulation of catechin biosynthesis in tea (Camellia sinensis). PLoS One. 2017:12(2):e0171173.

77. Fan K, Fan D, Ding Z, Su Y, Wang X. Cs-miR156 is involved in the nitrogen form regulation of catechins accumulation in tea plant (Camellia sinensis L.). Plant Physiol Biochem. 2015;97:350-60.

78. Cui L, Shan J, Shi M, Gao J, Lin H. The miR156-SPL9-DFR pathway coordinates the relationship between development and abiotic stress tolerance in plants. Plant J Cell Mol Biol. 2014;80(6):1108-17.

79. Reyes $J$, Chua NH. ABA induction of miR159 controls transcript levels of two MYB factors during Arabidopsis seed germination. Plant J. 2007:49(4):592-606

80. Allen RS, Li JY, Alonso-Peral MM, White RG, Guble F, Millar AA. MicroR159 regulation of most conserved targets in Arabidopsis has negligible phenotypic effects. Silence. 2010;1(1):18.

81. Abe H, Urao T, Ito T, Seki M, Shinozaki K, Yamaguchi-Shinozaki K. Arabidopsis AtMYC2 (bHLH) and AtMYB2 (MYB) function as transcriptional activators in abscisic acid signaling. Plant Cell. 2003;15(1):63-78.

82. Boualem A, Laporte P, Jovanovic M, Laffont C, Plet J, Combier JP, Niebel A Crespi M, Frugier F. MicroRNA166 controls root and nodule development in Medicago truncatula. Plant J. 2008;54(5):876-87.

83 Williams L, Grigg SP, Xie M, Christensen S, Fletcher JC. Regulation of Arabidopsis shoot apical meristem and lateral organ formation by microRNA miR166g and its AtHD-ZIP target genes. Development. 2005;132(16):3657-68.

84. Malamy JE. Intrinsic and environmental response pathways that regulate root system architecture. Plant Cell Environ. 2005;28(1):67-77.

85. Kawashima CG, Matthewman CA, Huang S, Lee BR, Yoshimoto N, Koprivova A, Rubio-Somoza I, Todesco M, Rathjen T, Saito K, Takahashi H, Dalmay T, Kopriva S. Interplay of SLIM1 and miR395 in the regulation of sulfate assimilation in Arabidopsis. Plant J. 2011;66(5):863-76.

86. Gallardo K, Courty PE, Le SC, Wipf D, Vernoud V. Sulfate transporters in the plant's response to drought and salinity: regulation and possible functions. Front Plant Sci. 2014;5:580.

87. Wang Y, Lin L, Sha T, Liu J, Zhang H, Hui Z, Jia G, Diao X. Combined small RNA and degradome sequencing to identify miRNAs and their targets in response to drought in foxtail millet. BMC Genet. 2016;17(1):57.

88. Leustek T, Murillo M, Cervantes M. Cloning of a cDNA encoding ATP sulfurylase from Arabidopsis thaliana by functional expression in Saccharomyces Cerevisiae. Plant Physiol. 1994;105(3):897-902.
89. Zhu L, Deng W, Ye A, Yu M, Wang Z, Jiang C. Cloning of two cDNAs encoding a family of ATP sulfurylase from Camellia sinensis related to selenium or sulfur metabolism and functional expression in Escherichia coli. Plant Physiol Biochem. 2008;46(9):731-8.

90. Mugford SG, Yoshimoto N, Reichelt M, Wirtz M, Hill L, Mugford ST, Nakazato Y, Noji M, Takahashi H, Kramell R, Gigolashvili T, Flügge UI, Wasternack C, Gershenzon J, Hell R, Saito K, Kopriva S. Disruption of Adenosine-5'Phosphosulfate Kinase in Arabidopsis reduces levels of sulfated secondary metabolites. Plant Cell. 2009;21(3):910.

91. Zholkevich VN, Zubkova NK, Maevskaya SN, Volkov VS, Rakitin VY, Kuznetsov W. Interaction between heat shock and water stress in plants: 2. Osmoregulation in the leaves of cotton plants successively treated with short-term hyperthermia and soil drought. Russ J Plant Physiol. 1997;44(4): 533-42.

92. Xia J, Zeng C, Chen Z, Zhang K, Chen X, Zhou YF, Song S, Lu C, Yang RJ, Yang Z, Zhou JF, Peng H, Wang WQ, Peng M, Zhang WX. Endogenous small-noncoding RNAs and their roles in chilling response and stress acclimation in cassava. BMC Genomics. 2014;15(1):634.

93. Reddy AR, Chaitanya KV, Vivekanandan M. Drought-induced responses of photosynthesis and antioxidant metabolism in higher plants. J Plant Physiol. 2004;161(11):1189-202.

94. Urano K, Kurihara Y, Seki M, Shinozaki K. Omics' analyses of regulatory networks in plant abiotic stress responses. Curr Opin Plant Biol. 2010; 13(2):132-8.

95. Jaleel CA, Manivannan P, Wahid A, Farooq M, Aljuburi H, Somasundaram R, Panneerselvam R. Drought stress in plants: a review on morphological characteristics and pigments composition. Int J Agric Biol. 2009;11(1):100-5.

96. Cruz de Carvalho MH. Drought stress and reactive oxygen species: production, scavenging and signaling. Plant Signal Behav. 2008;3(3):156-65.

\section{Submit your next manuscript to BioMed Central and we will help you at every step:}

- We accept pre-submission inquiries

- Our selector tool helps you to find the most relevant journal

- We provide round the clock customer support

- Convenient online submission

- Thorough peer review

- Inclusion in PubMed and all major indexing services

- Maximum visibility for your research

Submit your manuscript at www.biomedcentral.com/submit
) Biomed Central 